\title{
Green's function representations for seismic interferometry
}

\author{
Kees Wapenaar ${ }^{1}$ and Jacob Fokkema ${ }^{1}$
}

\begin{abstract}
The term seismic interferometry refers to the principle of generating new seismic responses by crosscorrelating seismic observations at different receiver locations. The first version of this principle was derived by Claerbout (1968), who showed that the reflection response of a horizontally layered medium can be synthesized from the autocorrelation of its transmission response. For an arbitrary 3D inhomogeneous lossless medium it follows from Rayleigh's reciprocity theorem and the principle of time-reversal invariance that the acoustic Green's function between any two points in the medium can be represented by an integral of crosscorrelations of wavefield observations at those two points. The integral is along sources on an arbitrarily shaped surface enclosing these points. No assumptions are made with respect to the diffusivity of the wavefield. The Rayleigh-Betti reciprocity theorem leads to a similar representation of the elastodynamic Green's function. When a part of the enclosing surface is the earth's free surface, the integral needs only to be evaluated over the remaining part of the closed surface. In practice, not all sources are equally important: The main contributions to the reconstructed Green's function come from sources at stationary points. When the sources emit transient signals, a shaping filter can be applied to correct for the differences in source wavelets. When the sources are uncorrelated noise sources, the representation simplifies to a direct crosscorrelation of wavefield observations at two points, similar as in methods that retrieve Green's functions from diffuse wavefields in disordered media or in finite media with an irregular bounding surface.
\end{abstract}

\section{INTRODUCTION}

It has been shown by many authors that the crosscorrelation of two recordings of a diffuse wavefield at two receiver positions leads to the Green's function that would be observed at one of these receiver positions if there were an impulsive source at the other. The diffusivity of the wavefield can be due to a random distribution of uncorrelated noise sources (Weaver and Lobkis, 2001, 2002; Wapenaar et al., 2002, 2004b; Shapiro and Campillo, 2004; Shapiro et al., 2005; Roux et al., 2005), reverberations in an enclosure with an irregular bounding surface (Lobkis and Weaver, 2001), multiple scattering between heterogeneities in a disordered medium (Campillo and Paul, 2003; Derode et al., 2003a; van Tiggelen, 2003; Malcolm et al., 2004; Snieder, 2004), or any combination of these causes.

Diffusivity of the wavefield is not a necessary condition for the retrieval of the Green's function by means of correlation. Claerbout (1968) showed that the autocorrelation of the transmission response of an arbitrary horizontally layered lossless earth yields its reflection response. This result has been generalized for three-dimensional inhomogeneous media by the authors (Wapenaar et al., 2002, 2004b). It appeared that the reflection response between a source and receiver at two positions at the earth's free surface can be expressed as an integral of crosscorrelations of transmission responses observed at the same two surface positions; the integral is along sources at some subsurface level. Since the reflection response of a medium relates downgoing to upgoing waves, it can be seen as the Green's function of the coupled one-way wave equations for downgoing and upgoing waves. In the above mentioned papers the relation between the reflection response and the correlation of the transmission responses was derived from a reciprocity theorem for the one-way wave equations. For this derivation it was not necessary to make any assumptions about the diffusivity of the wavefield. In the same papers we also derived a variant of the relation for the situation of uncorrelated noise sources in the subsurface (hence, for a specific type of diffuse wavefield). This led to a direct relation between the reflection response and the crosscorrelation of the transmission responses, without the integral along the sources. With the latter relation we confirmed a conjecture of Claerbout for the 3D situation. Following Schuster (2001), we use the term seismic interferometry for the process of generating new seismic responses by crosscorrelating seismic observations at different receiver locations.

Since a reflection response is the Green's function of the one-way

Manuscript received by the Editor May 12, 2005; revised manuscript received July 27, 2005; published online August 17, 2006.

${ }^{1}$ Delft University of Technology, Department of Geotechnology, Mijnbouwstraat 120, 2628 RX Delft, The Netherlands. E-mail: c.p.a.wapenaar@tudelft.nl; j.t.fokkema@tudelft.nl.

(C) 2006 Society of Exploration Geophysicists. All rights reserved. 
wave equations, it is quite natural to employ one-way wave theory for the derivation of interferometric relations. In one of the abovementioned papers (Wapenaar et al., 2004b), we presented an extensive overview of relations between reflection and transmission responses of 3D inhomogeneous media, based on reciprocity theorems for the one-way wave equations. However, these theorems also involve some restrictions with respect to the configuration. The main underlying assumption is that the boundary of the considered domain consists of two parallel horizontal surfaces, one of them usually coinciding with the earth's surface and the other being some arbitrary horizontal subsurface level (in general, not coinciding with a physical boundary). Although in practice this assumption can be somewhat relaxed, it implies a restriction with respect to the applications in seismic interferometry. Another complication of the oneway interferometric relations is that they apply to downgoing and upgoing wavefields. Hence, wavefield decomposition is required prior to employing one-way interferometry.

In the current paper we give an overview of representations of Green's functions in terms of crosscorrelations of full wavefields in arbitrary configurations (Wapenaar, 2004; Weaver and Lobkis, 2004; van Manen et al., 2005) and discuss modifications for their application in seismic interferometry. Note that the term Green's function is often associated with a solution of the wave equation for an impulsive point source in a background medium. Throughout this paper, however, we mean by Green's function the response of an impulsive point source in the actual medium. Similar to our derivations of the relations between the reflection and transmission responses we make no assumptions with respect to the diffusivity of the wavefield; the situation with uncorrelated noise sources is handled as a special case. We consider the acoustic as well as the elastodynamic situation. The paper is set up in such a way that the sections on the elastodynamic representations can be read independently from those on the acoustic representations.

\section{ACOUSTIC RECIPROCITY THEOREMS}

A reciprocity theorem relates two independent acoustic states in one and the same domain (de Hoop, 1988; Fokkema and van den Berg, 1993). Consider an acoustic wavefield, characterized by the acoustic pressure $p(\mathbf{x}, t)$ and the particle velocity $v_{i}(\mathbf{x}, t)$. Lower-case Latin subscripts take on the values 1, 2 and 3; furthermore, $\mathbf{x}$ $=\left(x_{1}, x_{2}, x_{3}\right)$ denotes the Cartesian coordinate vector (as usual the $x_{3}$-axis is pointing downward) and $t$ denotes time. We define the temporal Fourier transform of a space- and time-dependent quantity $p(\mathbf{x}, t)$ as

$$
\hat{p}(\mathbf{x}, \omega)=\int_{-\infty}^{\infty} \exp (-j \omega t) p(\mathbf{x}, t) \mathrm{d} t
$$

where $j$ is the imaginary unit and $\omega$ the angular frequency. In the space-frequency domain, the acoustic pressure and particle velocity in a lossless arbitrary inhomogeneous fluid medium obey the equation of motion

$$
j \omega \rho \hat{v}_{i}+\partial_{i} \hat{p}=\hat{f}_{i}
$$

and the stress-strain relation

$$
j \omega \kappa \hat{p}+\partial_{i} \hat{v}_{i}=\hat{q}
$$

Here $\partial_{i}$ denotes the partial derivative in the $x_{i}$-direction (Einstein's summation convention applies for repeated subscripts), $\rho(\mathbf{x})$ is the mass density of the medium, $\kappa(\mathbf{x})$ its compressibility, $\hat{f}_{i}(\mathbf{x}, \omega)$ the external volume force density, and $\hat{q}(\mathbf{x}, \omega)$ a source distribution in terms of volume injection rate density. We consider the interaction quantity (de Hoop, 1988)

$$
\partial_{i}\left\{\hat{p}_{A} \hat{v}_{i, B}-\hat{v}_{i, A} \hat{p}_{B}\right\},
$$

where subscripts $A$ and $B$ are used to distinguish two independent acoustic states. Rayleigh's reciprocity theorem is obtained by substituting the equation of motion (equation 2) and the stress-strain relation (equation 3 ) for states $A$ and $B$ into the interaction quantity (equation 4 ), integrating the result over an arbitrary spatial domain $\mathbb{D}$ enclosed by boundary $\partial \mathbb{D}$ with outward pointing normal vector $\mathbf{n}$ $=\left(n_{1}, n_{2}, n_{3}\right)$, and applying the theorem of Gauss. This gives

$$
\begin{gathered}
\int_{\mathrm{D}}\left\{\hat{p}_{A} \hat{q}_{B}-\hat{v}_{i, A} \hat{f}_{i, B}-\hat{q}_{A} \hat{p}_{B}+\hat{f}_{i, A} \hat{v}_{i, B}\right\} \mathrm{d}^{3} \mathbf{x} \\
=\oint_{\partial \mathrm{D}}\left\{\hat{p}_{A} \hat{v}_{i, B}-\hat{v}_{i, A} \hat{p}_{B}\right\} n_{i} \mathrm{~d}^{2} \mathbf{x}
\end{gathered}
$$

(Rayleigh, 1878; de Hoop, 1988; Fokkema and van den Berg, 1993). We call this a reciprocity theorem of the convolution type since the products in the frequency domain $\left(\hat{p}_{A} \hat{v}_{i, B}\right.$, etc. $)$ correspond to convolutions in the time domain.

Because the medium is assumed to be lossless, we can apply the principle of time-reversal invariance (Bojarski, 1983; Fink, 1997). In the frequency domain, time-reversal is replaced by complex conjugation. Hence, when $\hat{p}$ and $\hat{v}_{i}$ are a solution of the equation of motion and the stress-strain relation with source terms $\hat{f}_{i}$ and $\hat{q}$, then $\hat{p}^{*}$ and $-\hat{v}_{i}^{*}$ obey the same equations with source terms $\hat{f}_{i}^{*}$ and $-\hat{q}^{*}$ (the asterisk denotes complex conjugation). Making these substitutions for state $A$ we obtain

$$
\begin{gathered}
\int_{\mathrm{D}}\left\{\hat{p}_{A}^{*} \hat{q}_{B}+\hat{v}_{i, A}^{*} \hat{f}_{i, B}+\hat{q}_{A}^{*} \hat{p}_{B}+\hat{f}_{i, A}^{*} \hat{v}_{i, B}\right\} \mathrm{d}^{3} \mathbf{x} \\
=\oint_{\partial \mathrm{D}}\left\{\hat{p}_{A}^{*} \hat{v}_{i, B}+\hat{v}_{i, A}^{*} \hat{p}_{B}\right\} n_{i} \mathrm{~d}^{2} \mathbf{x} .
\end{gathered}
$$

We call this a reciprocity theorem of the correlation type because the products in the frequency domain $\left(\hat{p}_{A}^{*} \hat{v}_{i, B}\right.$, etc. $)$ correspond to correlations in the time domain.

Note that for both theorems we assumed that the medium parameters in states $A$ and $B$ are identical. de Hoop (1988) and Fokkema and van den Berg (1993) discuss more general reciprocity theorems that account also for different medium parameters in the two states.

\section{ACOUSTIC GREEN'S FUNCTION REPRESENTATIONS}

\section{Open configuration}

In this section, we substitute Green's functions for the wavefields in both acoustic reciprocity theorems. We show that the reciprocity theorem of the convolution type (equation 5) thus leads to the wellknown acoustic source-receiver reciprocity relation, whereas the reciprocity theorem of the correlation type (equation 6) yields acoustic Green's function representations, which are the basis for seismic 
interferometry. We consider an open configuration. The domain $\mathbb{D}$ with boundary $\partial \mathrm{D}$ is a subdomain of this open configuration; the boundary $\partial \mathrm{D}$ does in general not coincide with a physical boundary.

We choose impulsive point sources of volume injection rate in both states, according to

$$
\begin{aligned}
& q_{A}(\mathbf{x}, t)=\delta\left(\mathbf{x}-\mathbf{x}_{A}\right) \delta(t), \\
& q_{B}(\mathbf{x}, t)=\delta\left(\mathbf{x}-\mathbf{x}_{B}\right) \delta(t),
\end{aligned}
$$

or, in the frequency domain,

$$
\begin{aligned}
& \hat{q}_{A}(\mathbf{x}, \omega)=\delta\left(\mathbf{x}-\mathbf{x}_{A}\right), \\
& \hat{q}_{B}(\mathbf{x}, \omega)=\delta\left(\mathbf{x}-\mathbf{x}_{B}\right),
\end{aligned}
$$

with $\mathbf{x}_{A}$ and $\mathbf{x}_{B}$ both in $\mathbb{D}$; the external forces are chosen equal to zero in both states. The wavefields in states $A$ and $B$ can thus be expressed in terms of acoustic Green's functions, according to

$$
\begin{aligned}
\hat{p}_{A}(\mathbf{x}, \omega) & \triangleq \hat{G}\left(\mathbf{x}, \mathbf{x}_{A}, \omega\right), \\
\hat{v}_{i, A}(\mathbf{x}, \omega) & =-(j \omega \rho(\mathbf{x}))^{-1} \partial_{i} \hat{G}\left(\mathbf{x}, \mathbf{x}_{A}, \omega\right), \\
\hat{p}_{B}(\mathbf{x}, \omega) & \triangleq \hat{G}\left(\mathbf{x}, \mathbf{x}_{B}, \omega\right), \\
\hat{v}_{i, B}(\mathbf{x}, \omega) & =-(j \omega \rho(\mathbf{x}))^{-1} \partial_{i} \hat{G}\left(\mathbf{x}, \mathbf{x}_{B}, \omega\right) .
\end{aligned}
$$

The Green's function $\hat{G}\left(\mathbf{x}, \mathbf{x}_{A}, \omega\right)$ is the Fourier transform of the causal time-domain Green's function $G\left(\mathbf{x}, \mathbf{x}_{A}, t\right)$, which represents an impulse response observed at $\mathbf{x}$, due to a source at $\mathbf{x}_{A}$. According to equation 11, the observed wavefield quantity at $\mathbf{x}$ is acoustic pressure; according to equation 9 , the source at $\mathbf{x}_{A}$ is a volume injection rate source. Similar remarks hold for $\hat{G}\left(\mathbf{x}, \mathbf{x}_{B}, \omega\right)$. Other choices for the observed quantity at $\mathbf{x}$ and the source type at $\mathbf{x}_{A}$ and $\mathbf{x}_{B}$ are possible but will not be considered here because we prefer to keep the notation for the acoustic Green's functions simple. In the sections on the elastodynamic Green's function representations, we employ a modified notation that accounts for different observed wavefield quantities and different source types. Acoustic representations for Green's functions in terms of observed particle velocities and force sources can be obtained as a special case of the elastodynamic representations.

By substituting equations 9,11 , and 12 into equation 3 , it follows that $\hat{G}\left(\mathbf{x}, \mathbf{x}_{A}, \omega\right)$ obeys the wave equation

$$
\partial_{i}\left(\rho^{-1} \partial_{i} \hat{G}\right)+\left(\omega^{2} / \rho c^{2}\right) \hat{G}=-j \omega \delta\left(\mathbf{x}-\mathbf{x}_{A}\right),
$$

with propagation velocity $c(\mathbf{x})=\{\kappa(\mathbf{x}) \rho(\mathbf{x})\}^{-1 / 2}$. A similar wave equation holds for $\hat{G}\left(\mathbf{x}, \mathbf{x}_{B}, \omega\right)$.

Substituting equations 9-14 into the acoustic reciprocity theorem of the convolution type (equation 5) gives

$$
\begin{aligned}
\hat{G}\left(\mathbf{x}_{B}, \mathbf{x}_{A}, \omega\right)-\hat{G}\left(\mathbf{x}_{A}, \mathbf{x}_{B}, \omega\right) & \\
= & \oint_{\partial \mathrm{D}} \frac{-1}{j \omega \rho(\mathbf{x})}\left(\hat{G}\left(\mathbf{x}, \mathbf{x}_{A}, \omega\right) \partial_{i} \hat{G}\left(\mathbf{x}, \mathbf{x}_{B}, \omega\right)\right. \\
& \left.-\left(\partial_{i} \hat{G}\left(\mathbf{x}, \mathbf{x}_{A}, \omega\right)\right) \hat{G}\left(\mathbf{x}, \mathbf{x}_{B}, \omega\right)\right) n_{i} \mathrm{~d}^{2} \mathbf{x}
\end{aligned}
$$

Recall that the Green's functions $\hat{G}\left(\mathbf{x}, \mathbf{x}_{A}, \omega\right)$ and $\hat{G}\left(\mathbf{x}, \mathbf{x}_{B}, \omega\right)$ are the Fourier transforms of causal time-domain Green's functions. Hence, when $\partial \mathbb{D}$ is a spherical surface with infinite radius, then the right- hand side of equation 16 vanishes on account of the radiation conditions of the Green's functions (e.g., Bleistein, 1984). Moreover, since the right-hand side of equation 16 is independent of how $\partial \mathrm{D}$ is chosen (as long as it encloses $\mathbf{x}_{A}$ and $\mathbf{x}_{B}$ ), it vanishes for any $\partial \mathbb{D}$. Equation 16 thus yields

$$
\hat{G}\left(\mathbf{x}_{B}, \mathbf{x}_{A}, \omega\right)=\hat{G}\left(\mathbf{x}_{A}, \mathbf{x}_{B}, \omega\right) .
$$

This is the well-known source-receiver reciprocity relation for the acoustic Green's function.

Substituting equations 9-14 into the acoustic reciprocity theorem of the correlation type (equation 6) gives

$$
\begin{aligned}
\hat{G}^{*}\left(\mathbf{x}_{B}, \mathbf{x}_{A}, \omega\right)+\hat{G}\left(\mathbf{x}_{A}, \mathbf{x}_{B}, \omega\right) & \\
= & \oint_{\partial \mathrm{D}} \frac{-1}{j \omega \rho(\mathbf{x})}\left(\hat{G}^{*}\left(\mathbf{x}, \mathbf{x}_{A}, \omega\right) \partial_{i} \hat{G}\left(\mathbf{x}, \mathbf{x}_{B}, \omega\right)\right. \\
& \left.-\left(\partial_{i} \hat{G}^{*}\left(\mathbf{x}, \mathbf{x}_{A}, \omega\right)\right) \hat{G}\left(\mathbf{x}, \mathbf{x}_{B}, \omega\right)\right) n_{i} \mathrm{~d}^{2} \mathbf{x} .
\end{aligned}
$$

Again, the right-hand side is independent of the choice of $\partial \mathbb{D}$, as long as it encloses $\mathbf{x}_{A}$ and $\mathbf{x}_{B}$. Note, however, that since $\hat{G}^{*}\left(\mathbf{x}, \mathbf{x}_{A}, \omega\right)$ is the Fourier transform of the anticausal time-domain Green's function $G\left(\mathbf{x}, \mathbf{x}_{A},-t\right)$, the radiation conditions are not fulfilled and hence the right-hand side of equation 18 does not vanish. Using source-receiver reciprocity of the Green's functions gives

$$
\begin{aligned}
2 \Re\left\{\hat{G}\left(\mathbf{x}_{A}, \mathbf{x}_{B}, \omega\right)\right\}= & \oint_{\partial \mathrm{D}} \frac{-1}{j \omega \rho(\mathbf{x})}\left(\hat{G}^{*}\left(\mathbf{x}_{A}, \mathbf{x}, \omega\right) \partial_{i} \hat{G}\left(\mathbf{x}_{B}, \mathbf{x}, \omega\right)\right. \\
& \left.-\left(\partial_{i} \hat{G}^{*}\left(\mathbf{x}_{A}, \mathbf{x}, \omega\right)\right) \hat{G}\left(\mathbf{x}_{B}, \mathbf{x}, \omega\right)\right) n_{i} \mathrm{~d}^{2} \mathbf{x},
\end{aligned}
$$

where $\mathfrak{R}$ denotes the real part. Equation 19 is the basis for acoustic seismic interferometry, as will be discussed in a later section; van Manen et al. (2005) propose an efficient modeling scheme, based on an expression similar to equation 19.

The terms $\hat{G}$ and $\partial_{i} \hat{G} n_{i}$ under the integral in the right-hand side of equation 19 represent responses of monopole and dipole sources at $\mathbf{x}$ on $\partial \mathrm{D}$. The products $\hat{G}^{*} \partial_{i} \hat{G} n_{i}$, etc., correspond to crosscorrelations in the time domain. Hence, the right-hand side can be interpreted as the integral of the Fourier transform of crosscorrelations of observations of wavefields at $\mathbf{x}_{A}$ and $\mathbf{x}_{B}$, respectively, because of impulsive sources at $\mathbf{x}$ on $\partial \mathbb{D}$; the integration takes place along the source coordinate $\mathbf{x}$ (see Figure 1). The left-hand side of equation 19 is the Fourier transform of $G\left(\mathbf{x}_{A}, \mathbf{x}_{B}, t\right)+G\left(\mathbf{x}_{A}, \mathbf{x}_{B},-t\right)$, which is the superposition of the response at $\mathbf{x}_{A}$ due to an impulsive source at $\mathbf{x}_{B}$ and its time-reversed version. Because the Green's function $G\left(\mathbf{x}_{A}, \mathbf{x}_{B}, t\right)$ is causal, it can be obtained by taking the causal part of this superposition (or, more precisely, by multiplying this superposition with the Heaviside step function). Alternatively, in the frequency domain the imaginary part of $\hat{G}\left(\mathbf{x}_{A}, \mathbf{x}_{B}, \omega\right)$ can be obtained from the Hilbert transform of the real part.

Note that equation 19 is exact and applies to any lossless arbitrary inhomogeneous fluid medium. The choice of the integration boundary $\partial \mathrm{D}$ is arbitrary (as long as it encloses $\mathbf{x}_{A}$ and $\mathbf{x}_{B}$ ) and the medium may be inhomogeneous inside as well as outside $\partial \mathbb{D}$. The reconstructed Green's function $\hat{G}\left(\mathbf{x}_{A}, \mathbf{x}_{B}, \omega\right)$ contains, apart from the direct 
wave between $\mathbf{x}_{B}$ and $\mathbf{x}_{A}$, all scattering contributions (primaries and multiples) from inhomogeneities inside as well as outside $\partial \mathrm{D}$.

\section{Modified Green's function}

In many papers on Green's function retrieval, the imaginary part, instead of the real part of the function, is obtained. Here, we show that with a slight modification of the Green's function, we obtain a representation for the imaginary part of the Green's function instead of equation 19.

The Green's function $\hat{G}\left(\mathbf{x}, \mathbf{x}_{A}, \omega\right)$ represents the acoustic pressure due to a point source of volume injection rate (see equations 9 and 11). This Green's function obeys equation 15 , with the source term in the right-hand side defined as $-j \omega \delta\left(\mathbf{x}-\mathbf{x}_{A}\right)$. Let us define a new Green's function $\hat{\mathcal{G}}\left(\mathbf{x}, \mathbf{x}_{A}, \omega\right)$ representing the acoustic pressure due to a point source of volume injection (instead of volume injection rate). This Green's function obeys the same wave equation, but with the source in the right-hand side replaced by $-\delta\left(\mathbf{x}-\mathbf{x}_{A}\right)$, according to

$$
\partial_{i}\left(\rho^{-1} \partial_{i} \hat{\mathcal{G}}\right)+\left(\omega^{2} / \rho c^{2}\right) \hat{\mathcal{G}}=-\delta\left(\mathbf{x}-\mathbf{x}_{A}\right)
$$

A similar wave equation holds for $\hat{\mathcal{G}}\left(\mathbf{x}, \mathbf{x}_{B}, \omega\right)$. Note that $\hat{\mathcal{G}}$ and $\hat{G}$ are mutually related via $\hat{\mathcal{G}}=(1 / j \hat{\omega}) \hat{G}$. Following the same derivation as above, we obtain instead of equation 19

$$
\begin{aligned}
2 j \Im\left\{\hat{\mathcal{G}}\left(\mathbf{x}_{A}, \mathbf{x}_{B}, \omega\right)\right\}= & \oint_{\partial \mathrm{D}} \frac{1}{\rho(\mathbf{x})}\left(\hat{\mathcal{G}}^{*}\left(\mathbf{x}_{A}, \mathbf{x}, \omega\right) \partial_{i} \hat{\mathcal{G}}\left(\mathbf{x}_{B}, \mathbf{x}, \omega\right)\right. \\
& \left.-\left(\partial_{i} \hat{\mathcal{G}}^{*}\left(\mathbf{x}_{A}, \mathbf{x}, \omega\right)\right) \hat{\mathcal{G}}\left(\mathbf{x}_{B}, \mathbf{x}, \omega\right)\right) n_{i} \mathrm{~d}^{2} \mathbf{x},
\end{aligned}
$$

where $\mathfrak{I}$ denotes the imaginary part. The left-hand side of equation 21 is the Fourier transform of $\mathcal{G}\left(\mathbf{x}_{A}, \mathbf{x}_{B}, t\right)-\mathcal{G}\left(\mathbf{x}_{A}, \mathbf{x}_{B},-t\right)$, which is the difference of the response at $\mathbf{x}_{A}$ due to an impulsive source at $\mathbf{x}_{B}$ and its time-reversed version. Since the Green's function $\mathcal{G}\left(\mathbf{x}_{A}, \mathbf{x}_{B}, t\right)$ is causal, it can be obtained by taking the causal part of this difference. Alternatively, in the frequency domain the real part of $\hat{\mathcal{G}}\left(\mathbf{x}_{A}, \mathbf{x}_{B}, \omega\right)$ can be obtained from the Hilbert transform of the imaginary part.

Because $2 j \Im\{\hat{\mathcal{G}}\}=\frac{1}{j \omega} 2 \mathfrak{R}\{\hat{G}\}$, equation 21 does not provide new information in comparison with equation 19 ; it only serves as a link

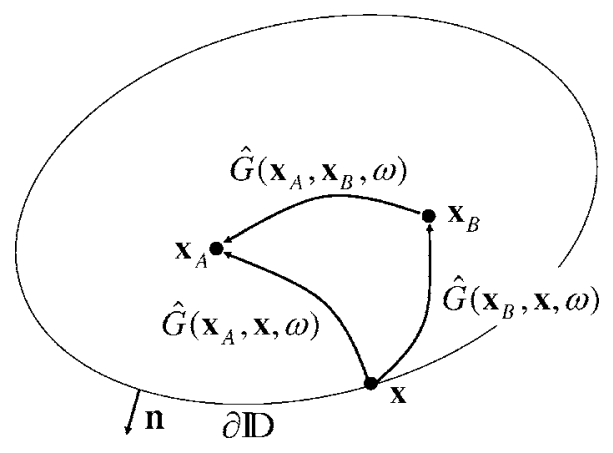

Figure 1. According to equation 19 , the Green's function $\hat{G}\left(\mathbf{x}_{A}\right.$, $\left.\mathbf{x}_{B}, \omega\right)$ can be obtained by crosscorrelating observations at $\mathbf{x}_{A}$ and $\mathbf{x}_{B}$ and integrating along the source coordinate $\mathbf{x}$ at $\partial \mathrm{D}$. Note that the rays in this figure represent the full responses between the source and receiver points, including primary and multiple scattering due to inhomogeneities inside as well as outside $\partial \mathbb{D}$. with other literature on Green's function retrieval (see the remarks below equation 32).

\section{Configuration with a free surface}

We consider a modified configuration for which we define the closed surface as $\partial \mathrm{D}=\partial \mathrm{D}_{0} \cup \partial \mathrm{D}_{1}$, where $\partial \mathbb{D}_{0}$ is a part of the earth's free surface and $\partial \mathrm{D}_{1}$ an arbitrarily shaped surface, in general not coinciding with a physical boundary. We consider the situation for which $\mathbf{x}_{A}$ and $\mathbf{x}_{B}$ are located inside $\partial \mathrm{D}_{0} \cup \partial \mathrm{D}_{1}$ (see Figure 2). For this configuration, we can use the results of the previous sections. Because the acoustic pressure $\hat{p}$ vanishes on $\partial \mathbb{D}_{0}$, the integral on the right-hand side of equations $6,18,19$, and 21 needs only be evaluated over $\partial \mathrm{D}_{1}$. Hence, the Green's function $\hat{G}\left(\mathbf{x}_{A}, \mathbf{x}_{B}, \omega\right)$ or $\hat{\mathcal{G}}\left(\mathbf{x}_{A}, \mathbf{x}_{B}, \omega\right)$ can be recovered by crosscorrelating and integrating the responses of sources on $\partial \mathrm{D}_{1}$ only.

\section{MODIFICATIONS FOR ACOUSTIC SEISMIC INTERFEROMETRY}

Equation 19 is an exact representation of the acoustic Green's function, but in its present form it is not very well suited for application in seismic interferometry. The main complication is that the integrand consists of a superposition of two correlation products that need to be evaluated separately. Moreover, monopole as well as dipole responses are assumed to be available for all source positions $\mathbf{x}$ on $\partial \mathrm{D}$. Finally, the sources are assumed to be impulsive point sources, which does not comply with reality. In this section, we first discuss a number of simplifications of the integrand of equation 19. Next we discuss the modifications of equation 19 for realistic sources (transient as well as noise sources).

\section{Simplification of the integrand}

In the following, we first investigate the effect of scatterers outside the integration boundary $\partial \mathbb{D}$. Next we discuss the approximations that are needed so that the integrand of equation 19 reduces to a single correlation product. Finally we discuss the approximation that is required when only monopole responses are available.

The starting point for the analysis in this section is equation 19, with integration boundary $\partial \mathbb{D}$ (see Figure 1). However, everything that is discussed below also applies to the free surface configuration of Figure 2, with integration boundary $\partial \mathrm{D}_{1}$. Moreover, all results below can be easily adapted for the modified Green's function $\hat{\mathcal{G}}$, simply by substituting $\hat{G}=j \omega \hat{\mathcal{G}}$.

We temporarily denote $\hat{G}\left(\mathbf{x}_{A}, \mathbf{x}, \omega\right)$ and $\hat{G}\left(\mathbf{x}_{B}, \mathbf{x}, \omega\right)$ by $\hat{G}_{A}$ and $\hat{G}_{B}$, respectively. Furthermore, we write

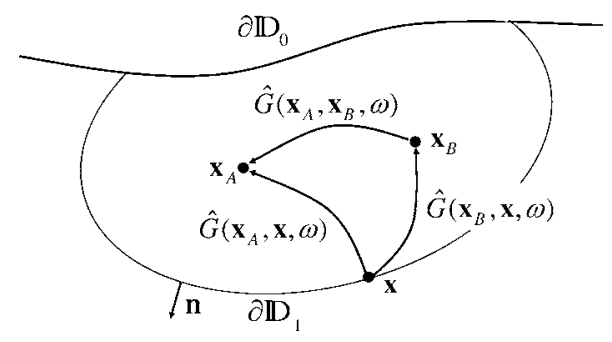

Figure 2. Modified configuration, with a free surface $\partial \mathrm{D}_{0}$. The rays represent the full responses, including primary and multiple scattering due to inhomogeneities inside as well as outside $\partial \mathbb{D}_{1}$ as well as reflections from the free surface $\partial \mathrm{D}_{0}$. 


$$
\begin{aligned}
& \hat{G}_{A}=\hat{G}_{A}^{\text {in }}+\hat{G}_{A}^{\text {out }}, \\
& \hat{G}_{B}=\hat{G}_{B}^{\text {in }}+\hat{G}_{B}^{\text {out }},
\end{aligned}
$$

where the superscripts in and out refer to waves propagating inward and outward from the sources at $\mathbf{x}$ on $\partial \mathrm{D}$ (see Figure 3 ). Substituting these expressions into equation 19 gives

$$
\begin{aligned}
2 \Re\{ & \left.\hat{G}\left(\mathbf{x}_{A}, \mathbf{x}_{B}, \omega\right)\right\} \\
= & \oint_{\partial \mathrm{D}} \frac{-1}{j \omega \rho(\mathbf{x})}\left(\left(\hat{G}_{A}^{\text {in* }}+\hat{G}_{A}^{\text {out**}}\right)\left(\partial_{i} \hat{G}_{B}^{\text {in }}+\partial_{i} \hat{G}_{B}^{\text {out }}\right)\right. \\
& \left.-\left(\partial_{i} \hat{G}_{A}^{\text {in* }}+\partial_{i} \hat{G}_{A}^{\text {out* }}\right)\left(\hat{G}_{B}^{\text {in }}+\hat{G}_{B}^{\text {out }}\right)\right) n_{i} \mathrm{~d}^{2} \mathbf{x} .
\end{aligned}
$$

Assuming the medium is smooth in a small region around $\partial \mathrm{D}$, the normal derivatives of the Green's functions can be approximated in the high frequency regime by multiplying each constituent (direct wave, scattered wave etc.) by $\mp j \frac{\omega}{c(\mathbf{x})}|\cos \alpha(\mathbf{x})|$, where $c(\mathbf{x})$ is the local propagation velocity at $\partial \mathrm{D}$ and $\alpha(\mathbf{x})$ the local angle between the pertinent ray and the normal on $\partial \mathrm{D}$. The minus-sign applies to inward propagating waves and the plus-sign to outward propagating waves. The main contributions to the integral in equation 24 come from stationary points on $\partial \mathrm{D}$ (Schuster et al., 2004; Wapenaar et al., 2004a; Snieder, 2004; Snieder et al., 2006). At those points the absolute cosines of the ray angles for $\hat{G}_{A}$ and $\hat{G}_{B}$ are identical. This implies, for example, that the terms $\hat{G}_{A}^{\text {in* }} \partial_{i} \hat{G}_{B}^{\text {in }} n_{i}$ and $-\left(\partial_{i} \hat{G}_{A}^{\text {in } *}\right) \hat{G}_{B}^{\text {in }} n_{i}$ give equal contributions to the integral, whereas the contributions of $\hat{G}_{A}^{\text {in* }} \partial_{i} \hat{G}_{B}^{\text {out }} n_{i}$ and $-\left(\partial_{i} \hat{G}_{A}^{\text {in* }}\right) \hat{G}_{B}^{\text {out }} n_{i}$ cancel each other. Hence, we can rewrite equation 24 as

$$
\begin{aligned}
2 \Re\{ & \left.\hat{G}\left(\mathbf{x}_{A}, \mathbf{x}_{B}, \omega\right)\right\} \\
& =\oint_{\partial \mathrm{D}} \frac{2}{j \omega \rho(\mathbf{x})}\left(\left(\partial_{i} \hat{G}_{A}^{\text {in* }}\right) \hat{G}_{B}^{\text {in }}+\left(\partial_{i} \hat{G}_{A}^{\text {out }}\right) \hat{G}_{B}^{\text {out }}\right) n_{i} \mathrm{~d}^{2} \mathbf{x} .
\end{aligned}
$$

Of course, the inward and outward propagating waves at $\mathbf{x}$ on $\partial \mathbb{D}$ cannot be separately measured at $\mathbf{x}_{A}$ and $\mathbf{x}_{B}$. We use equations 22 and 23 to rewrite the integrand of equation 25 as

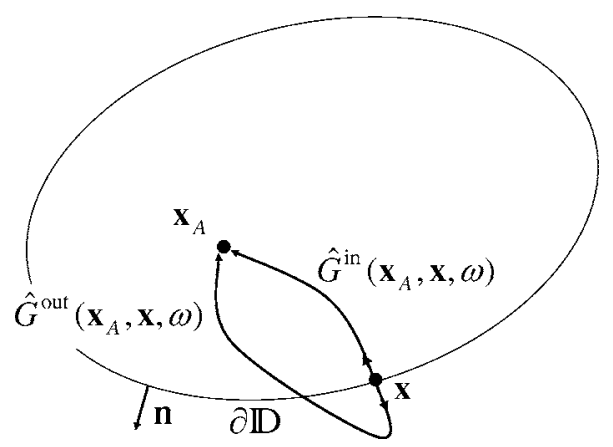

Figure 3. When the medium outside $\partial \mathbb{D}$ is inhomogeneous, then the Green's function $\hat{G}\left(\mathbf{x}_{A}, \mathbf{x}, \omega\right)$ consists of a term $\hat{G}^{\text {in }}\left(\mathbf{x}_{A}, \mathbf{x}, \omega\right)$ that propagates inward from the source at $\mathbf{x}$ on $\partial \mathrm{D}$ to $\mathbf{x}_{A}$ and a term $\hat{G}^{\text {out }}\left(\mathbf{x}_{A}, \mathbf{x}, \omega\right)$ that propagates outward from the source at $\mathbf{x}$ on $\partial \mathbb{D}$ and reaches $\mathbf{x}_{A}$ after having been scattered at inhomogeneities outside $\partial \mathrm{D}$. In the text these terms are abbreviated as $\hat{G}_{A}^{\text {in }}$ and $\hat{G}_{A}^{\text {out }}$, respectively.

$$
\begin{aligned}
& \left(\partial_{i} \hat{G}_{A}^{\text {in* }}\right) \hat{G}_{B}^{\text {in }}+\left(\partial_{i} \hat{G}_{A}^{\text {out* }}\right) \hat{G}_{B}^{\text {out }} \\
& \quad=\left(\partial_{i} \hat{G}_{A}^{*}\right) \hat{G}_{B}-\left(\partial_{i} \hat{G}_{A}^{\text {in* }}\right) \hat{G}_{B}^{\text {out }}-\left(\partial_{i} \hat{G}_{A}^{\text {out* }}\right) \hat{G}_{B}^{\text {in }} .
\end{aligned}
$$

Substituting this into the right-hand side of equation 25 yields

$$
\begin{aligned}
& 2 \mathfrak{R}\left\{\hat{G}\left(\mathbf{x}_{A}, \mathbf{x}_{B}, \omega\right)\right\}+\text { 'ghost' } \\
& \quad=\oint_{\partial \mathrm{D}} \frac{2}{j \omega \rho(\mathbf{x})}\left(\partial_{i} \hat{G}^{*}\left(\mathbf{x}_{A}, \mathbf{x}, \omega\right)\right) \hat{G}\left(\mathbf{x}_{B}, \mathbf{x}, \omega\right) n_{i} \mathrm{~d}^{2} \mathbf{x},
\end{aligned}
$$

where

$$
\text { 'ghost' }=\oint_{\partial \mathrm{D}} \frac{2}{j \omega \rho(\mathbf{x})}\left(\left(\partial_{i} \hat{G}_{A}^{\mathrm{in} *}\right) \hat{G}_{B}^{\mathrm{out}}+\left(\partial_{i} \hat{G}_{A}^{\mathrm{out} *}\right) \hat{G}_{B}^{\mathrm{in}}\right) n_{i} \mathrm{~d}^{2} \mathbf{x} .
$$

The right-hand side of equation 27 contains only one correlation product and therefore has a more manageable form than equation 19. However, the left-hand side of equation 27 contains a ghost term that adds spurious events to the reconstructed Green's function $\hat{G}\left(\mathbf{x}_{A}, \mathbf{x}_{B}, \omega\right)$. According to equation 28 , this ghost term contains correlation products of waves that propagate inward in one state and outward in the other. Note that when $\partial \mathrm{D}$ is an irregular surface (which is the case when the sources are randomly distributed), these correlation products are not integrated coherently in equation 28 , and therefore their contribution can be ignored in equation 27 . Hence, the Green's function $\hat{G}\left(\mathbf{x}_{A}, \mathbf{x}_{B}, \omega\right)$ can be accurately retrieved from the right-hand side of equation 27 as long as $\partial \mathbb{D}$ is sufficiently irregular. The resulting reconstructed Green's function contains all scattering effects from inhomogeneities inside as well as outside $\partial \mathbb{D}$. This interesting phenomenon was first observed with numerical experiments by Draganov et al. $(2003,2006)$.

Recall that $\hat{G}_{A}^{\text {out }}$ stands for $\hat{G}^{\text {out }}\left(\mathbf{x}_{A}, \mathbf{x}, \omega\right)$, i.e., a Green's wavefield that propagates outward from the source at $\mathbf{x}$ on $\partial \mathrm{D}$, gets scattered at inhomogeneities outside $\partial \mathrm{D}$ and propagates to the observation point $\mathbf{x}_{A}$ inside $\partial \mathbb{D}$ (see Figure 3 ). A similar remark applies to $\hat{G}_{B}^{\text {out }}$. From here onward, we assume that the medium at and outside $\partial \mathbb{D}$ is homogeneous, with propagation velocity $c$ and mass density $\rho$, and that the Green's functions $\hat{G}_{A}^{\text {out }}$ and $\hat{G}_{B}^{\text {out }}$ are zero. This implies that the ghost term defined by equation 28 vanishes, hence

$$
\begin{aligned}
2 \Re\{ & \left.\hat{G}\left(\mathbf{x}_{A}, \mathbf{x}_{B}, \omega\right)\right\} \\
& =\frac{2}{j \omega \rho} \oint_{\partial \mathrm{D}}\left(\partial_{i} \hat{G}^{*}\left(\mathbf{x}_{A}, \mathbf{x}, \omega\right)\right) \hat{G}\left(\mathbf{x}_{B}, \mathbf{x}, \omega\right) n_{i} \mathrm{~d}^{2} \mathbf{x} .
\end{aligned}
$$

Despite the simple form of equation 29 in comparison with the original equation 19 (i.e., one correlation product instead of two), this equation still requires the availability of monopole- and dipolesource responses. When only monopole responses are available, we have to express the dipole response $\partial_{i} \hat{G}\left(\mathbf{x}_{A}, \mathbf{x}, \omega\right) n_{i}$ in terms of the monopole response $\hat{G}\left(\mathbf{x}_{A}, \mathbf{x}, \omega\right)$. As explained before, to this end each constituent of the monopole response should be multiplied by $-j \frac{\omega}{c}|\cos \alpha(\mathbf{x})|$, where $\alpha(\mathbf{x})$ is the local angle between the pertinent ray and the normal on $\partial \mathbb{D}$. However, since $\alpha(\mathbf{x})$ may have multiple values, and because these values are generally unknown (unless the 
inhomogeneous medium and source positions are known), we approximate the dipole response by

$$
\partial_{i} \hat{G}\left(\mathbf{x}_{A}, \mathbf{x}, \omega\right) n_{i} \approx-j \frac{\omega}{c} \hat{G}\left(\mathbf{x}_{A}, \mathbf{x}, \omega\right)
$$

hence

$$
2 \mathfrak{R}\left\{\hat{G}\left(\mathbf{x}_{A}, \mathbf{x}_{B}, \omega\right)\right\} \approx \frac{2}{\rho c} \oint_{\partial \mathrm{D}} \hat{G}^{*}\left(\mathbf{x}_{A}, \mathbf{x}, \omega\right) \hat{G}\left(\mathbf{x}_{B}, \mathbf{x}, \omega\right) \mathrm{d}^{2} \mathbf{x}
$$

The approximation in equation 30 is quite accurate when $\partial \mathrm{D}$ is a sphere with a very large radius, because in this case all rays are normal to $\partial \mathrm{D}$ (i.e., $\alpha \approx 0$ ). In general, however, this approximation involves an amplitude error that can be significant. Moreover, spurious events may occur due to incomplete cancellation of contributions from different stationary points. However, since the approximation in equation 30 does not affect the phase of equation 31, it is considered acceptable for seismic interferometry. Apart from the proportionality factor $2 / \rho c$, equation 31 was also obtained by Derode et al. (2003a,b) purely by physical reasoning.

Using the modified Green's function $\hat{\mathcal{G}}=\frac{1}{j \omega} \hat{G}$, we obtain instead of equation 31

$$
2 j \Im\left\{\hat{\mathcal{G}}\left(\mathbf{x}_{A}, \mathbf{x}_{B}, \omega\right)\right\} \approx-\frac{2 j \omega}{\rho c} \oint_{\partial \mathrm{D}} \hat{\mathcal{G}}^{*}\left(\mathbf{x}_{A}, \mathbf{x}, \omega\right) \hat{\mathcal{G}}\left(\mathbf{x}_{B}, \mathbf{x}, \omega\right) \mathrm{d}^{2} \mathbf{x}
$$

The left-hand side is the Fourier transform of $\mathcal{G}\left(\mathbf{x}_{A}, \mathbf{x}_{B}, t\right)-\mathcal{G}\left(\mathbf{x}_{A}, \mathbf{x}_{B}\right.$, $-t$ ); the factor $j \omega$ in the right-hand side corresponds to a differentiation in the time domain. Hence, equation 32 resembles the results of Weaver and Lobkis (2004) and Snieder (2004), who retrieve the antisymmetric two-sided Green's function from the time-derivative of crosscorrelations.

We summarize the assumptions and approximations that we have made in deriving equation 31 (or 32) from equation 19 (or 21). We made a high frequency approximation to reduce the integrand to a single correlation product (equation 27), we assumed that the medium at and outside $\partial \mathrm{D}$ is homogeneous to remove the ghost term (equation 29), and we assumed $\alpha \approx 0$ to replace the dipole response by a monopole response (equation 31 or 32 ).

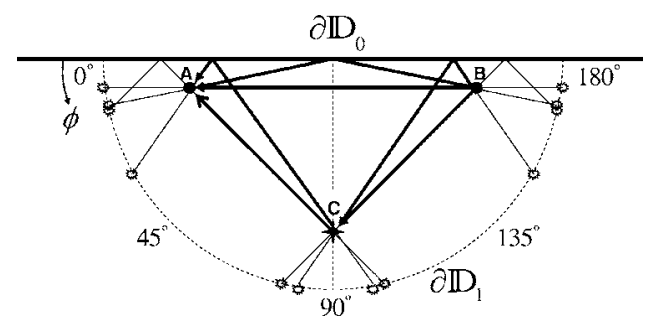

Figure 4. Single diffractor $(\mathrm{C})$ in a homogeneous medium below a free surface. The receivers are at $\mathrm{A}$ and $\mathrm{B}$. The numerical integration is carried out along the sources on the surface $\partial \mathbb{D}_{1}$. The causal contributions come from the indicated stationary points between $\phi=0^{\circ}$ and $45^{\circ}$, the anticausal contributions from the indicated points between $\phi=135^{\circ}$ and $180^{\circ}$. The contributions from the indicated stationary points around $\phi=90^{\circ}$ cancel each other.

\section{Numerical example}

We illustrate equation 29 with a $2 \mathrm{D}$ example for a configuration with a free surface at $x_{3}=0$. We consider a single diffractor at $\left(x_{1}, x_{3}\right)=(0,600) \mathrm{m}$ in a homogeneous medium with propagation velocity $c=2000 \mathrm{~m} / \mathrm{s}$ (see Figure 4 ), in which $\mathrm{C}$ denotes the diffractor. Further, we define $\mathbf{x}_{A}=(-500,100) \mathrm{m}$ and $\mathbf{x}_{B}=(500$, $100) \mathrm{m}$, denoted by $\mathrm{A}$ and $\mathrm{B}$ in Figure 4 . The surface $\partial \mathbb{D}_{1}$ is a semicircle with its center at the origin and a radius of $800 \mathrm{~m}$. The solid arrows in Figure 4 denote the Green's function $G\left(\mathbf{x}_{A}, \mathbf{x}_{B}, t\right)$. For the Green's functions in equation 29, we use analytical expressions based on the Born approximation (hence, the contrast at the point diffractor is assumed to be small). To be consistent with the Born approximation, in the crosscorrelations we consider only the zeroth and first order terms. Figure 5a shows the time-domain representation of the integrand of equation 29 (convolved with a wavelet with a central frequency of $50 \mathrm{~Hz}$ ). Each trace corresponds to a fixed source position $\mathbf{x}$ on $\partial \mathbb{D}_{1}$; the source position in polar coordinates is $(\phi, r=800)$. The sum of all these traces (multiplied by $r \mathrm{~d} \phi)$ is shown in Figure $5 \mathrm{~b}$. This result accurately matches the time-domain version of the left-hand side of equation 29 , i.e., $G\left(\mathbf{x}_{A}, \mathbf{x}_{B}, t\right)$ $+G\left(\mathbf{x}_{A}, \mathbf{x}_{B},-t\right)$, convolved with a wavelet (see Figure 6). Figure 5 clearly shows that the main contributions come from Fresnel zones around the stationary points of the integrand. The causal contributions come from the indicated stationary points in Figure 4 between $\phi=0^{\circ}$ and $45^{\circ}$, the anticausal contributions from the indicated points between $\phi=135^{\circ}$ and $180^{\circ}$. The contributions from the indicated stationary points around $\phi=90^{\circ}$ cancel each other.

\section{Transient sources}

Until now we assumed that the sources on $\partial \mathrm{D}$ are impulsive point sources. When the sources are transient sources with wavelet $s(\mathbf{x}, t)$ and corresponding spectrum $\hat{s}(\mathbf{x}, \omega)$, we write for the observed wavefields at $\mathbf{x}_{A}$ and $\mathbf{x}_{B}$

$$
\begin{aligned}
& \hat{p}^{\mathrm{obs}}\left(\mathbf{x}_{A}, \mathbf{x}, \omega\right)=\hat{G}\left(\mathbf{x}_{A}, \mathbf{x}, \omega\right) \hat{s}(\mathbf{x}, \omega), \\
& \hat{p}^{\mathrm{obs}}\left(\mathbf{x}_{B}, \mathbf{x}, \omega\right)=\hat{G}\left(\mathbf{x}_{B}, \mathbf{x}, \omega\right) \hat{s}(\mathbf{x}, \omega) .
\end{aligned}
$$

We define the power spectrum of the sources as

$$
\hat{S}(\mathbf{x}, \omega)=\hat{s}^{*}(\mathbf{x}, \omega) \hat{s}(\mathbf{x}, \omega) .
$$

Using these equations, we can modify equation 31 as follows

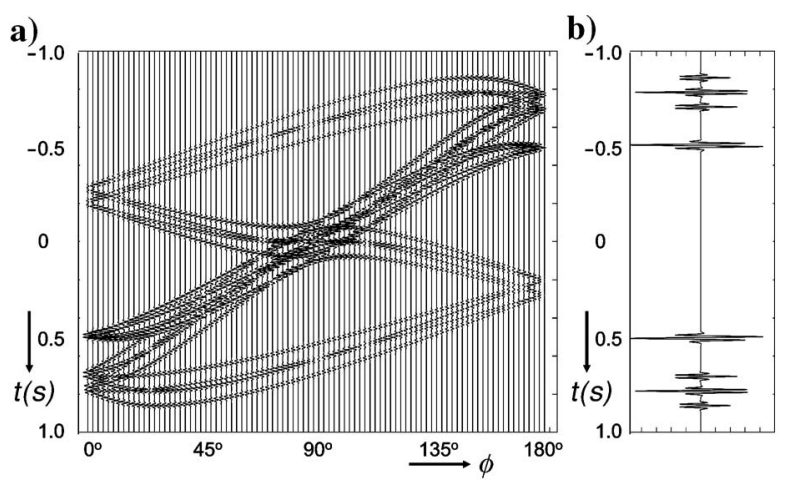

Figure 5. (a) Time domain representation of the integrand of equation 29. (b) The sum of all traces in (a). 


$$
\begin{aligned}
2 \mathfrak{R}\left\{\hat{G}\left(\mathbf{x}_{A}, \mathbf{x}_{B}, \omega\right)\right\} \hat{S}_{0}(\omega) \\
\quad \approx \frac{2}{\rho c} \oint_{\partial \mathrm{D}} \hat{\mathcal{F}}(\mathbf{x}, \omega) \hat{p}^{\text {obs* }}\left(\mathbf{x}_{A}, \mathbf{x}, \omega\right) \hat{p}^{\mathrm{obs}}\left(\mathbf{x}_{B}, \mathbf{x}, \omega\right) \mathrm{d}^{2} \mathbf{x},
\end{aligned}
$$

where $\hat{S}_{0}(\omega)$ is some average (arbitrarily chosen) power spectrum and $\hat{\mathcal{F}}(\mathbf{x}, \omega)$ is a shaping filter defined as

$$
\hat{\mathcal{F}}(\mathbf{x}, \omega)=\frac{\hat{S}_{0}(\omega)}{\hat{S}(\mathbf{x}, \omega)} .
$$

Equation 36 is well suited for seismic interferometry. It can be applied when a number of natural transient sources with different wavelets on $\partial \mathrm{D}$ radiate wavefields to $\mathbf{x}_{A}$ and $\mathbf{x}_{B}$, that are measured independently for each source at $\mathbf{x}$ on $\partial \mathrm{D}$. The shaping filter corrects for the differences in the power spectra of the different sources on $\partial \mathrm{D}$ (this requires that these power spectra are known). Not all sources are equally important; the main contributions to the reconstructed Green's function come from stationary points on $\partial \mathrm{D}$, as was illustrated with the numerical example. For a careful stationary phase analysis of seismic interferometry, see Snieder et al. (2006).

\section{Uncorrelated noise sources}

For the transient sources discussed above, we had to assume that the response of each source at $\mathbf{x}$ on $\partial \mathrm{D}$ could be measured separately. Here we show that this need is obviated when the sources are mutually uncorrelated noise sources (Weaver and Lobkis, 2001, 2002; Wapenaar et al., 2002, 2004b; Derode et al., 2003a; Weaver and Lobkis, 2004; Snieder, 2004; Roux et al., 2005; Shapiro et al., 2005). We define the noise signal at $\mathbf{x}$ on $\partial \mathbb{D}$ as $N(\mathbf{x}, t)$ and its corresponding spectrum as $\hat{N}(\mathbf{x}, \omega)$. When all noise sources act simultaneously, we may write for the observed wavefields at $\mathbf{x}_{A}$ and $\mathbf{x}_{B}$

$$
\begin{aligned}
& \hat{p}^{\mathrm{obs}}\left(\mathbf{x}_{A}, \omega\right)=\oint_{\partial \mathrm{D}} \hat{G}\left(\mathbf{x}_{A}, \mathbf{x}, \omega\right) \hat{N}(\mathbf{x}, \omega) \mathrm{d}^{2} \mathbf{x} \\
& \hat{p}^{\mathrm{obs}}\left(\mathbf{x}_{B}, \omega\right)=\oint_{\partial \mathrm{D}} \hat{G}\left(\mathbf{x}_{B}, \mathbf{x}^{\prime}, \omega\right) \hat{N}\left(\mathbf{x}^{\prime}, \omega\right) \mathrm{d}^{2} \mathbf{x}^{\prime}
\end{aligned}
$$

We assume that two noise sources $\hat{N}(\mathbf{x}, \omega)$ and $\hat{N}\left(\mathbf{x}^{\prime}, \omega\right)$ are mutually uncorrelated for any $\mathbf{x} \neq \mathbf{x}^{\prime}$ at $\partial \mathrm{D}$, and that their power spectrum is the same for all $\mathbf{x}$. Hence, we assume that these noise sources obey the relation

$$
\left\langle\hat{N}^{*}(\mathbf{x}, \omega) \hat{N}\left(\mathbf{x}^{\prime}, \omega\right)\right\rangle=\delta\left(\mathbf{x}-\mathbf{x}^{\prime}\right) \hat{S}(\omega)
$$

where $\langle\cdot\rangle$ denotes a spatial ensemble average and $\hat{S}(\omega)$ the power spectrum of the noise. Evaluating the crosscorrelation of the observed wavefields $\hat{p}^{\text {obs }}\left(\mathbf{x}_{A}, \omega\right)$ and $\hat{p}^{\text {obs }}\left(\mathbf{x}_{B}, \omega\right)$, using equations $38-40$, yields

$$
\begin{aligned}
\left\langle\hat{p}^{\text {obs* }}\left(\mathbf{x}_{A}, \omega\right) \hat{p}^{\mathrm{obs}}\left(\mathbf{x}_{B}, \omega\right)\right\rangle \\
=\oint_{\partial \mathrm{D}} \hat{G}^{*}\left(\mathbf{x}_{A}, \mathbf{x}, \omega\right) \hat{G}\left(\mathbf{x}_{B}, \mathbf{x}, \omega\right) \hat{S}(\omega) \mathrm{d}^{2} \mathbf{x} .
\end{aligned}
$$

Combining this with equation 31 , we obtain

$$
2 \Re\left\{\hat{G}\left(\mathbf{x}_{A}, \mathbf{x}_{B}, \omega\right)\right\} \hat{S}(\omega) \approx \frac{2}{\rho c}\left\langle\hat{p}^{\mathrm{obs} *}\left(\mathbf{x}_{A}, \omega\right) \hat{p}^{\mathrm{obs}}\left(\mathbf{x}_{B}, \omega\right)\right\rangle .
$$

Equation 42 is well suited for application in seismic interferometry. The advantage of equation 42 over equation 36 is that no separate measurements of the responses of all sources at $\partial \mathrm{D}$ are required; these responses can be measured simultaneously, according to equations 38 and 39. The disadvantage is that no corrections can be made for different power spectra of different sources, like with the shaping filter $\hat{\mathcal{F}}(\mathbf{x}, \omega)$ in equation 36 .

Finally, note that in the time domain equation 42 becomes

$$
\begin{aligned}
& \int_{-\infty}^{\infty}\left\{G\left(\mathbf{x}_{A}, \mathbf{x}_{B}, t^{\prime}\right)+G\left(\mathbf{x}_{A}, \mathbf{x}_{B},-t^{\prime}\right)\right\} S\left(t-t^{\prime}\right) \mathrm{d} t^{\prime} \\
& \approx \frac{2}{\rho c}\left\langle\int_{-\infty}^{\infty} p^{\text {obs }}\left(\mathbf{x}_{A}, t^{\prime}\right) p^{\text {obs }}\left(\mathbf{x}_{B}, t+t^{\prime}\right) \mathrm{d} t^{\prime}\right) .
\end{aligned}
$$

According to this equation, the crosscorrelation of the observed pressures at $\mathbf{x}_{A}$ and $\mathbf{x}_{B}$ yields the Green's function for a receiver at $\mathbf{x}_{A}$ and a source at $\mathbf{x}_{B}$, convolved with the autocorrelation of the noise sources. Note the striking resemblance with the retrieval of the Green's function in diffuse wavefields in finite media with an irregular bounding surface or in disordered media, as discussed by Lobkis and Weaver (2001), van Tiggelen (2003), Malcolm et al. (2004), and Snieder (2004).

\section{ELASTODYNAMIC RECIPROCITY THEOREMS}

Consider an elastodynamic wavefield, characterized by the stress tensor $\tau_{i j}(\mathbf{x}, t)$ and the particle velocity $v_{i}(\mathbf{x}, t)$. In the space-frequency domain, the stress tensor and particle velocity in a lossless arbitrary inhomogeneous anisotropic solid medium obey the equation of motion

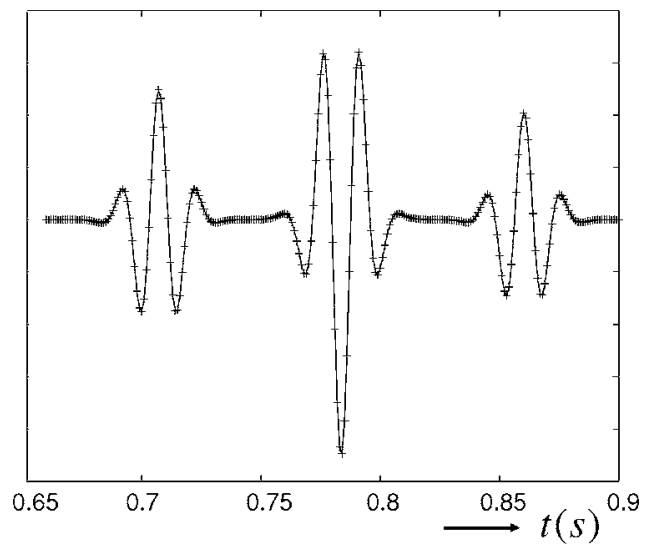

Figure 6. Zoomed-in version of the causal scattered events in Figure $5 \mathrm{~b}$. The solid line is the time-domain version of the left-hand side of equation 29 . The plus-signs $(+)$ represent the numerical integration result of the right-hand side of equation 29 (i.e., the sum of the traces in Figure 5a). 


$$
j \omega \rho \hat{v}_{i}-\partial_{j} \hat{\tau}_{i j}=\hat{f}_{i}
$$

and the stress-strain relation

$$
-j \omega s_{i j k l} \hat{\tau}_{k l}+\left(\partial_{j} \hat{v}_{i}+\partial_{i} \hat{v}_{j}\right) / 2=\hat{h}_{i j},
$$

where $\rho(\mathbf{x})$ is the mass density of the medium, $s_{i j k l}(\mathbf{x})$ its compliance, $\hat{f}_{i}(\mathbf{x}, \omega)$ the external volume force density, and $\hat{h}_{i j}(\mathbf{x}, \omega)$ the external deformation rate density. We consider the interaction quantity

$$
\partial_{j}\left\{\hat{v}_{i, A} \hat{\tau}_{i j, B}-\hat{\tau}_{i j, A} \hat{v}_{i, B}\right\}
$$

where subscripts $A$ and $B$ are used to distinguish two independent elastodynamic states. The Rayleigh-Betti reciprocity theorem is obtained by substituting the equation of motion (equation 44) and the stress-strain relation (equation 45 ) for states $A$ and $B$ into the interaction quantity (equation 46), using the symmetry relations $\hat{\tau}_{i j}=\hat{\tau}_{j i}$ and $s_{i j k l}=s_{k l i j}$, integrating the result over an arbitrary spatial domain $\mathrm{D}$ enclosed by boundary $\partial \mathrm{D}$ with outward pointing normal vector $\mathbf{n}$ $=\left(n_{1}, n_{2}, n_{3}\right)$, and applying the theorem of Gauss. This gives

$$
\begin{gathered}
\int_{\mathbb{D}}\left\{-\hat{\tau}_{i j, A} \hat{h}_{i j, B}-\hat{v}_{i, A} \hat{f}_{i, B}+\hat{h}_{i j, A} \hat{\tau}_{i j, B}+\hat{f}_{i, A} \hat{v}_{i, B}\right\} \mathrm{d}^{3} \mathbf{x} \\
=\oint_{\partial \mathrm{D}}\left\{\hat{v}_{i, A} \hat{\tau}_{i j, B}-\hat{\tau}_{i j, A} \hat{v}_{i, B}\right\} n_{j} \mathrm{~d}^{2} \mathbf{x}
\end{gathered}
$$

(Knopoff and Gangi, 1959; de Hoop, 1966; Aki and Richards, 1980). This is the elastodynamic reciprocity theorem of the convolution type.

Because the medium is assumed to be lossless, we can apply the principle of time-reversal invariance (Bojarski, 1983). Hence, when $\hat{\tau}_{i j}$ and $\hat{v}_{i}$ are a solution of the equation of motion and the stress-strain relation with source terms $\hat{f}_{i}$ and $\hat{h}_{i j}$, then $\hat{\tau}_{i j}^{*}$ and $-\hat{v}_{i}^{*}$ obey the same equations with source terms $\hat{f}_{i}^{*}$ and $-\hat{h}_{i j}^{*}$. Making these substitutions for state $A$, we obtain

$$
\begin{gathered}
\int_{\mathbb{D}}\left\{-\hat{\tau}_{i j, A}^{*} \hat{h}_{i j, B}+\hat{v}_{i, A}^{*} \hat{f}_{i, B}-\hat{h}_{i j, A}^{*} \hat{\tau}_{i j, B}+\hat{f}_{i, A}^{*} \hat{v}_{i, B}\right\} \mathrm{d}^{3} \mathbf{x} \\
=\oint_{\partial \mathbb{D}}\left\{-\hat{v}_{i, A}^{*} \hat{\tau}_{i j, B}-\hat{\tau}_{i j, A}^{*} \hat{v}_{i, B}\right\} n_{j} \mathrm{~d}^{2} \mathbf{x} .
\end{gathered}
$$

This is the elastodynamic reciprocity theorem of the correlation type.

Note that for both theorems we assumed that the medium parameters in states $A$ and $B$ are identical. de Hoop (1995) discusses more general reciprocity theorems that account also for different medium parameters in the two states.

\section{ELASTODYNAMIC GREEN'S FUNCTION REPRESENTATIONS}

\section{Open configuration}

In this section, we substitute Green's functions for the wavefields in both elastodynamic reciprocity theorems. Thus, we show that the reciprocity theorem of the convolution type (equation 47) leads to the well-known elastodynamic source-receiver reciprocity relation, whereas the reciprocity theorem of the correlation type (equation 48) yields elastodynamic Green's function representations, which are the basis for seismic interferometry. We consider an open configura- tion. The domain $\mathrm{D}$ with boundary $\partial \mathrm{D}$ is a subdomain of this open configuration; the boundary $\partial \mathrm{D}$ in general does not coincide with a physical boundary.

We choose impulsive point sources of force in both states, according to

$$
\begin{aligned}
& f_{i, A}(\mathbf{x}, t)=\delta\left(\mathbf{x}-\mathbf{x}_{A}\right) \delta(t) \delta_{i p}, \\
& f_{i, B}(\mathbf{x}, t)=\delta\left(\mathbf{x}-\mathbf{x}_{B}\right) \delta(t) \delta_{i q},
\end{aligned}
$$

or, in the frequency domain,

$$
\begin{gathered}
\hat{f}_{i, A}(\mathbf{x}, \omega)=\delta\left(\mathbf{x}-\mathbf{x}_{A}\right) \delta_{i p}, \\
\hat{f}_{i, B}(\mathbf{x}, \omega)=\delta\left(\mathbf{x}-\mathbf{x}_{B}\right) \delta_{i q},
\end{gathered}
$$

with $\mathbf{x}_{A}$ and $\mathbf{x}_{B}$ both in $\mathrm{D}$; the deformation sources are chosen equal to zero in both states. The wavefields in states $A$ and $B$ can thus be expressed in terms of elastodynamic Green's functions, according to

$$
\begin{aligned}
\hat{v}_{i, A}(\mathbf{x}, \omega) & \triangleq \hat{G}_{i, p}^{v, f}\left(\mathbf{x}, \mathbf{x}_{A}, \omega\right), \\
\hat{\tau}_{i j, A}(\mathbf{x}, \omega) & =(j \omega)^{-1} c_{i j k l}(\mathbf{x}) \partial_{l} \hat{G}_{k, p}^{v, f}\left(\mathbf{x}, \mathbf{x}_{A}, \omega\right) \\
& \triangleq \hat{G}_{i j, p}^{\tau, f}\left(\mathbf{x}, \mathbf{x}_{A}, \omega\right), \\
\hat{v}_{i, B}(\mathbf{x}, \omega) & \triangleq \hat{G}_{i, q}^{v, f}\left(\mathbf{x}, \mathbf{x}_{B}, \omega\right), \\
\hat{\tau}_{i j, B}(\mathbf{x}, \omega) & =(j \omega)^{-1} c_{i j k l}(\mathbf{x}) \partial_{l} \hat{G}_{k, q}^{v, f}\left(\mathbf{x}, \mathbf{x}_{B}, \omega\right) \\
& \triangleq \hat{G}_{i j, q}^{\tau, f}\left(\mathbf{x}, \mathbf{x}_{B}, \omega\right),
\end{aligned}
$$

where the stiffness $c_{i j k l}$ is the inverse of the compliance $s_{i j k l}$, according to

$$
c_{i j k l} s_{k l m n}=s_{i j k l} c_{k l m n}=\frac{1}{2}\left(\delta_{i m} \delta_{j n}+\delta_{i n} \delta_{j m}\right) .
$$

We explain the notation convention for the elastodynamic Green's functions at the hand of $\hat{G}_{i, p}^{v, f}\left(\mathbf{x}, \mathbf{x}_{A}, \omega\right)$. This Green's function is the Fourier transform of the causal time-domain Green's function $G_{i, p}^{v, f}\left(\mathbf{x}, \mathbf{x}_{A}, t\right)$, which represents an impulse response observed at $\mathbf{x}$, due to a source at $\mathbf{x}_{A}$. The superscripts (here $v$ and $f$ ) represent the observed quantity (particle velocity) and the source quantity (force), respectively; the subscripts (here $i$ and $p$ ) represent the components of the observed quantity and the source quantity, respectively.

Substituting equations 51, 53, and 54 into equation 44, it follows that $\hat{G}_{i, p}^{v, f}\left(\mathbf{x}, \mathbf{x}_{A}, \omega\right)$ obeys the wave equation

$$
\partial_{j}\left(c_{i j k l} \partial_{l} \hat{G}_{k, p}^{v, f}\right)+\rho \omega^{2} \hat{G}_{i, p}^{v, f}=-j \omega \delta\left(\mathbf{x}-\mathbf{x}_{A}\right) \delta_{i p} .
$$

A similar wave equation holds for $\hat{G}_{i, q}^{v, f}\left(\mathbf{x}, \mathbf{x}_{B}, \omega\right)$.

Substituting equations 51-56 into the elastodynamic reciprocity theorem of the convolution type (equation 47) gives

$$
\begin{aligned}
-\hat{G}_{q, p}^{v, f}\left(\mathbf{x}_{B}, \mathbf{x}_{A}, \omega\right)+\hat{G}_{p, q}^{v, f}\left(\mathbf{x}_{A}, \mathbf{x}_{B}, \omega\right) \\
=\oint_{\partial \mathrm{D}}\left(\hat{G}_{i, p}^{v, f}\left(\mathbf{x}, \mathbf{x}_{A}, \omega\right) \hat{G}_{i j, q}^{\tau, f}\left(\mathbf{x}, \mathbf{x}_{B}, \omega\right)\right. \\
\left.\quad-\hat{G}_{i j, p}^{\tau, f}\left(\mathbf{x}, \mathbf{x}_{A}, \omega\right) \hat{G}_{i, q}^{v, f}\left(\mathbf{x}, \mathbf{x}_{B}, \omega\right)\right) n_{j} \mathrm{~d}^{2} \mathbf{x} .
\end{aligned}
$$


Recall that the Green's functions are the Fourier transforms of causal time-domain Green's functions. Hence, when $\partial \mathrm{D}$ is a spherical surface with infinite radius, then the right-hand side of equation 59 vanishes on account of the radiation conditions of the Green's functions (e.g., Pao and Varatharajulu, 1976). Moreover, since the right-hand side of equation 59 is independent of how $\partial \mathrm{D}$ is chosen (as long as it encloses $\mathbf{x}_{A}$ and $\mathbf{x}_{B}$ ), it vanishes for any $\partial \mathrm{D}$. Equation 59 thus yields

$$
\hat{G}_{q, p}^{v, f}\left(\mathbf{x}_{B}, \mathbf{x}_{A}, \omega\right)=\hat{G}_{p, q}^{v, f}\left(\mathbf{x}_{A}, \mathbf{x}_{B}, \omega\right) .
$$

This is the well-known source-receiver reciprocity relation for the elastodynamic Green's function.

If we replace the source in equation 52 by a point source of the deformation type, according to $\hat{h}_{i j, B}(\mathbf{x}, \omega)=\delta\left(\mathbf{x}-\mathbf{x}_{B}\right) \delta_{i q} \delta_{j r}$, then a similar derivation as above yields the following source-receiver reciprocity relation

$$
\hat{G}_{q r, p}^{\tau, f}\left(\mathbf{x}_{B}, \mathbf{x}_{A}, \omega\right)=\hat{G}_{p, q r}^{v, h}\left(\mathbf{x}_{A}, \mathbf{x}_{B}, \omega\right) .
$$

Substituting equations 51-56 into the elastodynamic reciprocity theorem of the correlation type (equation 48) gives

$$
\begin{aligned}
\left\{\hat{G}_{q, p}^{v, f}\left(\mathbf{x}_{B}, \mathbf{x}_{A}, \omega\right)\right\}^{*}+\hat{G}_{p, q}^{v, f}\left(\mathbf{x}_{A}, \mathbf{x}_{B}, \omega\right) & \\
= & -\oint_{\partial \mathrm{D}}\left(\left\{\hat{G}_{i, p}^{v, f}\left(\mathbf{x}, \mathbf{x}_{A}, \omega\right)\right\}^{*} \hat{G}_{i j, q}^{\tau, f}\left(\mathbf{x}, \mathbf{x}_{B}, \omega\right)\right. \\
& \left.+\left\{\hat{G}_{i j, p}^{\tau, f}\left(\mathbf{x}, \mathbf{x}_{A}, \omega\right)\right\}^{*} \hat{G}_{i, q}^{v, f}\left(\mathbf{x}, \mathbf{x}_{B}, \omega\right)\right) n_{j} \mathrm{~d}^{2} \mathbf{x} .
\end{aligned}
$$

Again, the right-hand side is independent of the choice of $\partial \mathrm{D}$, as long as it encloses $\mathbf{x}_{A}$ and $\mathbf{x}_{B}$. Note, however, that since $\left\{\hat{G}_{i, p}^{v, f}\left(\mathbf{x}, \mathbf{x}_{A}, \omega\right)\right\}^{*}$ and $\left\{\hat{G}_{i j, p}^{\tau, f}\left(\mathbf{x}, \mathbf{x}_{A}, \omega\right)\right\}^{*}$ are the Fourier transforms of the anticausal time-domain Green's functions $G_{i, p}^{v, f}\left(\mathbf{x}, \mathbf{x}_{A},-t\right)$ and $G_{i j, p}^{\tau f}\left(\mathbf{x}, \mathbf{x}_{A},-t\right)$, the radiation conditions are not fulfilled and hence the right-hand side of equation 62 does not vanish. Using source-receiver reciprocity of the Green's functions gives

$$
\begin{aligned}
2 \Re\left\{\hat{G}_{p, q}^{v, f}\left(\mathbf{x}_{A}, \mathbf{x}_{B}, \omega\right)\right\}= & -\oint_{\partial \mathrm{D}}\left(\left\{\hat{G}_{p, i}^{v, f}\left(\mathbf{x}_{A}, \mathbf{x}, \omega\right)\right\}^{*} \hat{G}_{q, i j}^{v, h}\left(\mathbf{x}_{B}, \mathbf{x}, \omega\right)\right. \\
& \left.+\left\{\hat{G}_{p, i j}^{v, h}\left(\mathbf{x}_{A}, \mathbf{x}, \omega\right)\right\}^{*} \hat{G}_{q, i}^{v, f}\left(\mathbf{x}_{B}, \mathbf{x}, \omega\right)\right) n_{j} \mathrm{~d}^{2} \mathbf{x}
\end{aligned}
$$

(Wapenaar, 2004). Equation 63 is the basis for elastodynamic seismic interferometry, as will be discussed in a later section.

The terms $\hat{G}_{p, i}^{v, f}$ and $\hat{G}_{q, i, j}^{v, h}$, under the integral in the right-hand side of equation 63, represent responses of force and deformation sources at $\mathbf{x}$ on $\partial \mathrm{D}$. The products $\left\{\hat{G}_{p, i}^{v, f}\right\}^{*} \hat{G}_{q, i, h}^{v,}$, etc., correspond to crosscorrelations in the time domain. Hence, the right-hand side can be interpreted as the integral of the Fourier transform of crosscorrelations of observations of wavefields at $\mathbf{x}_{A}$ and $\mathbf{x}_{B}$, respectively, due to impulsive sources at $\mathbf{x}$ on $\partial \mathrm{D}$; the integration takes place along the source coordinate $\mathbf{x}$ (see Figure 7). The left-hand side of equation 63 is the Fourier transform of $G_{p, q}^{v, f}\left(\mathbf{x}_{A}, \mathbf{x}_{B}, t\right)+G_{p, q}^{v, f}\left(\mathbf{x}_{A}, \mathbf{x}_{B},-t\right)$, which is the superposition of the response at $\mathbf{x}_{A}$ due to an impulsive source at $\mathbf{x}_{B}$ and its time-reversed version. Since the Green's function $G_{p, q}^{v, f}\left(\mathbf{x}_{A}, \mathbf{x}_{B}, t\right)$ is causal, it can be obtained by taking the causal part of this superposition (or, more precisely, by multiplying this superposition with the Heaviside step function). Alternatively, in the frequency domain the imaginary part of $\hat{G}_{p, q}^{v, f}\left(\mathbf{x}_{A}, \mathbf{x}_{B}, \omega\right)$ can be obtained from the Hilbert transform of the real part.
Note that equation 63 is exact and applies to any lossless arbitrary inhomogeneous anisotropic solid medium. The choice of the integration boundary $\partial \mathrm{D}$ is arbitrary (as long as it encloses $\mathbf{x}_{A}$ and $\mathbf{x}_{B}$ ) and the medium may be inhomogeneous and anisotropic inside as well as outside $\partial \mathrm{D}$. The reconstructed Green's function $\hat{G}_{p, q}^{v f f}\left(\mathbf{x}_{A}, \mathbf{x}_{B}, \omega\right)$ contains, apart from the direct wave between $\mathbf{x}_{B}$ and $\mathbf{x}_{A}$, all scattering contributions (primaries, multiples and mode conversions) from inhomogeneities inside as well as outside $\partial \mathrm{D}$.

\section{Modified Green's function}

The Green's function $\hat{G}_{i, p}^{v}\left(\mathbf{x}, \mathbf{x}_{A}, \omega\right)$ represents the particle velocity due to a point source of force (see equations 51 and 53). This Green's function obeys equation 58 , with the source term in the right-hand side defined as $-j \omega \delta\left(\mathbf{x}-\mathbf{x}_{A}\right) \delta_{i p}$. Let us define a new Green's function $\hat{G}_{i, p}^{u, f}\left(\mathbf{x}, \mathbf{x}_{A}, \omega\right)$, representing the particle displacement due to a point source of force. This Green's function obeys the same wave equation, but with the source in the right-hand side replaced by $-\delta\left(\mathbf{x}-\mathbf{x}_{A}\right) \delta_{i p}$, according to

$$
\partial_{j}\left(c_{i j k l} \partial_{l} \hat{G}_{k, p}^{u, f}\right)+\rho \omega^{2} \hat{G}_{i, p}^{u, f}=-\delta\left(\mathbf{x}-\mathbf{x}_{A}\right) \delta_{i p}
$$

A similar wave equation holds for $\hat{G}_{i, q}^{u, f}\left(\mathbf{x}, \mathbf{x}_{B}, \omega\right)$. Note that $\hat{G}_{i, p}^{u, f}$ and $\hat{G}_{i, p}^{v, f}$ are mutually related via $\hat{G}_{i, p}^{u, f}=\frac{1}{j \omega} \hat{G}_{i, p}^{v, f}$. Following the same derivation as above, we obtain instead of equation 63

$$
\begin{gathered}
2 j \Im\left\{\hat{G}_{p, q}^{u, f}\left(\mathbf{x}_{A}, \mathbf{x}_{B}, \omega\right)\right\}=j \omega \oint_{\partial \mathrm{D}}\left(\left\{\hat{G}_{p, i}^{u, f}\left(\mathbf{x}_{A}, \mathbf{x}, \omega\right)\right\}^{*} \hat{G}_{q, i j}^{u, h}\left(\mathbf{x}_{B}, \mathbf{x}, \omega\right)\right. \\
\left.+\left\{\hat{G}_{p, i j}^{u, h}\left(\mathbf{x}_{A}, \mathbf{x}, \omega\right)\right\}^{*} \hat{G}_{q, i}^{u, f}\left(\mathbf{x}_{B}, \mathbf{x}, \omega\right)\right) n_{j} \mathrm{~d}^{2} \mathbf{x}
\end{gathered}
$$

The left-hand side of equation 65 is the Fourier transform of $G_{p, q}^{u, f}\left(\mathbf{x}_{A}, \mathbf{x}_{B}, t\right)-G_{p, q}^{u, f}\left(\mathbf{x}_{A}, \mathbf{x}_{B},-t\right)$, which is the difference of the response at $\mathbf{x}_{A}$ due to an impulsive source at $\mathbf{x}_{B}$ and its time-reversed version. Because the Green's function $G_{p, q}^{u, f}\left(\mathbf{x}_{A}, \mathbf{x}_{B}, t\right)$ is causal, it can be obtained by taking the causal part of this difference. Alternatively, in the frequency domain the real part of $\hat{G}_{p, q}^{u, f}\left(\mathbf{x}_{A}, \mathbf{x}_{B}, \omega\right)$ can be obtained from the Hilbert transform of the imaginary part.

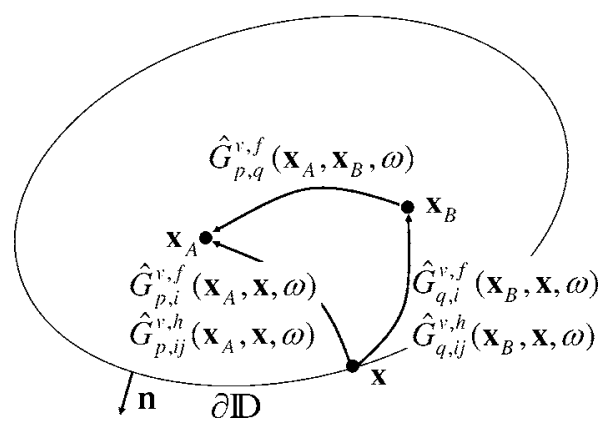

Figure 7. According to equation 63 , the Green's function $\hat{G}_{p, q}^{v, f}\left(\mathbf{x}_{A}\right.$, $\left.\mathbf{x}_{B}, \omega\right)$ can be obtained by crosscorrelating observations at $\mathbf{x}_{A}$ and $\mathbf{x}_{B}$ and integrating along the source coordinate $\mathbf{x}$ at $\partial \mathrm{D}$. Note that the rays in this figure represent the full responses between the source and receiver points, including primary and multiple scattering as well as mode conversion due to inhomogeneities inside as well as outside $\partial \mathrm{D}$. 


\section{Configuration with a free surface}

We consider a modified configuration for which we define the closed surface as $\partial \mathrm{D}=\partial \mathrm{D}_{0} \cup \partial \mathrm{D}_{1}$, where $\partial \mathrm{D}_{0}$ is a part of the earth's free surface and $\partial \mathrm{D}_{1}$ an arbitrarily shaped surface, in general not coinciding with a physical boundary. First, we consider the situation for which $\mathbf{x}_{A}$ and $\mathbf{x}_{B}$ are located inside $\partial \mathbb{D}_{0} \cup \partial \mathbb{D}_{1}$, similar as in Figure 2. For this configuration, we can use the results of the previous sections. Because the elastodynamic traction $\hat{\tau}_{i j} n_{j}$ vanishes on $\partial \mathrm{D}_{0}$, the integral on the right-hand side of equations $48,62,63$, and 65 needs only be evaluated over $\partial \mathrm{D}_{1}$. Hence, the Green's function $\hat{G}_{p, q}^{v, f}\left(\mathbf{x}_{A}, \mathbf{x}_{B}, \omega\right)$ or $\hat{G}_{p, q}^{u, f}\left(\mathbf{x}_{A}, \mathbf{x}_{B}, \omega\right)$ can be recovered by crosscorrelating and integrating the responses of sources on $\partial \mathrm{D}_{1}$ only.

Next, we consider the situation for which $\mathbf{x}_{A}$ and $\mathbf{x}_{B}$ are located at the free surface $\partial \mathrm{D}_{0}$ (see Figure 8). For this situation, we reconsider the elastodynamic reciprocity theorem of the correlation type (equation 48), in which we set the sources $\hat{f}_{i, A}, \hat{f}_{i, B}, \hat{h}_{i j, A}$, and $\hat{h}_{i j, B}$ in $\mathbb{D}$ equal to zero. Hence, the domain integral on the left-hand side of equation 48 vanishes. For the right-hand side of equation 48 , we separately consider the boundary integrals along $\partial \mathrm{D}_{0}$ and $\partial \mathbb{D}_{1}$, hence

$$
\begin{aligned}
\int_{\partial \mathrm{D}_{0}}\left\{\hat{v}_{i, A}^{*} \hat{\tau}_{i j, B}+\hat{\tau}_{i j, A}^{*} \hat{v}_{i, B}\right\} n_{j} \mathrm{~d}^{2} \mathbf{x} \\
\quad=-\int_{\partial \mathrm{D}_{1}}\left\{\hat{v}_{i, A}^{*} \hat{\tau}_{i j, B}+\hat{\tau}_{i j, A}^{*} \hat{v}_{i, B}\right\} n_{j} \mathrm{~d}^{2} \mathbf{x} .
\end{aligned}
$$

We introduce sources in terms of boundary conditions at the free surface $\partial \mathrm{D}_{0}$. This is possible because at a free surface the traction is zero everywhere, except at those positions where a source traction is applied. Hence, for $\mathbf{x} \in \partial \mathrm{D}_{0}$, the tractions in both states read

$$
\begin{aligned}
& \hat{\tau}_{i j, A}(\mathbf{x}, \omega) n_{j}=\delta\left(\mathbf{x}-\mathbf{x}_{A}\right) \delta_{i p}, \\
& \hat{\tau}_{i j, B}(\mathbf{x}, \omega) n_{j}=\delta\left(\mathbf{x}-\mathbf{x}_{B}\right) \delta_{i q},
\end{aligned}
$$

with $\mathbf{x}_{A}$ and $\mathbf{x}_{B}$ both at $\partial \mathrm{D}_{0}$. For the particle velocities at the free surface we write

$$
\begin{aligned}
& \hat{v}_{i, A}(\mathbf{x}, \omega) \triangleq \hat{G}_{i, p}^{v, \tau}\left(\mathbf{x}, \mathbf{x}_{A}, \omega\right), \\
& \hat{v}_{i, B}(\mathbf{x}, \omega) \triangleq \hat{G}_{i, q}^{v, \tau}\left(\mathbf{x}, \mathbf{x}_{B}, \omega\right),
\end{aligned}
$$

where the second superscript $\tau$ refers to the traction sources at $\mathbf{x}_{A}$ and $\mathbf{x}_{B}$. Substituting equations 67-70 into the left-hand side of equation 66 , and using the source-receiver reciprocity relation $\hat{G}_{q, p}^{v, \tau}\left(\mathbf{x}_{B}, \mathbf{x}_{A}, \omega\right)$ $=\hat{G}_{p, q}^{v, \tau}\left(\mathbf{x}_{A}, \mathbf{x}_{B}, \omega\right)$ gives

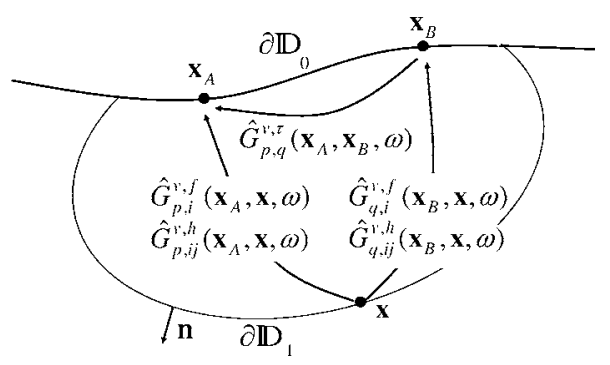

Figure 8. Modified configuration, with $\mathbf{x}_{A}$ and $\mathbf{x}_{B}$ at the free surface $\partial \mathbb{D}_{0}$. The rays represent again the full responses.

$$
\int_{\partial \mathrm{D}_{0}}\left\{\hat{v}_{i, A}^{*} \hat{\tau}_{i j, B}+\hat{\tau}_{i j, A}^{*} \hat{v}_{i, B}\right\} n_{j} \mathrm{~d}^{2} \mathbf{x}=2 \Re\left\{\hat{G}_{p, q}^{v, \tau}\left(\mathbf{x}_{A}, \mathbf{x}_{B}, \omega\right)\right\}
$$

In order to evaluate the right-hand side of equation 66, we express the wavefields at $\partial \mathrm{D}_{1}$ analogous to equations 53-56, but with the second superscript $f$ of all Green's functions replaced by $\tau$. Substituting these wavefields into the right-hand side of equation 66, applying the appropriate source-receiver relations, and combining the result with equation 71 for the left-hand side of equation 66 , we obtain

$$
\begin{aligned}
2 \mathfrak{R}\left\{\hat{G}_{p, q}^{v, \tau}\left(\mathbf{x}_{A}, \mathbf{x}_{B}, \omega\right)\right\} \\
=-\int_{\partial \mathrm{D}_{1}}\left(\left\{\hat{G}_{p, i}^{v, f}\left(\mathbf{x}_{A}, \mathbf{x}, \omega\right)\right\}^{*} \hat{G}_{q, i j}^{v, h}\left(\mathbf{x}_{B}, \mathbf{x}, \omega\right)\right. \\
\left.\quad+\left\{\hat{G}_{p, i j}^{v, h}\left(\mathbf{x}_{A}, \mathbf{x}, \omega\right)\right\}^{*} \hat{G}_{q, i}^{v, f}\left(\mathbf{x}_{B}, \mathbf{x}, \omega\right)\right) n_{j} \mathrm{~d}^{2} \mathbf{x} .
\end{aligned}
$$

Note the similarity with equation 63 . The various Green's functions in this representation are indicated in Figure 8. Using the modified Green's function $\hat{G}_{i, p}^{u, \tau}=\frac{1}{j \omega} \hat{G}_{i, p}^{v, \tau}$, etc., we obtain an expression similar to equation 65 .

\section{MODIFICATIONS FOR ELASTODYNAMIC SEISMIC INTERFEROMETRY}

Equation 63 (as well as equation 72) is an exact representation of the elastodynamic Green's function, but in its present form it is not very well suited for application in seismic interferometry. The main complication is that the integrand consists of a superposition of two correlation products that need to be evaluated separately. Moreover, force- as well as deformation-source responses are assumed to be available for all source positions $\mathbf{x}$ on $\partial \mathrm{D}$. Finally, the sources are assumed to be impulsive point sources, which does not comply with reality. In this section, we first discuss a simplification of the integrand of equation 63. Next, we discuss the modifications of equation 63 for realistic sources (transient as well as noise sources).

\section{Simplification of the integrand}

Unlike in the stepwise analysis of the integrand of the acoustic Green's function representation (equation 19), we straightaway assume that the medium at and outside $\partial \mathrm{D}$ is homogeneous and isotropic, with $\mathrm{P}$ - and $\mathrm{S}$-wave propagation velocities $c_{P}$ and $c_{S}$, respectively, and mass density $\rho$. In the Appendix, we show that for this situation equation 63 can be rewritten as

$$
\begin{aligned}
2 \Re\{ & \left.\hat{G}_{p, q}^{v, f}\left(\mathbf{x}_{A}, \mathbf{x}_{B}, \omega\right)\right\} \\
& =\frac{2}{j \omega \rho} \oint_{\partial \mathrm{D}}\left\{\partial_{i} \hat{G}_{p, K}^{v, \phi}\left(\mathbf{x}_{A}, \mathbf{x}, \omega\right)\right\}^{*} \hat{G}_{q, K}^{v, \phi}\left(\mathbf{x}_{B}, \mathbf{x}, \omega\right) n_{i} \mathrm{~d}^{2} \mathbf{x} .
\end{aligned}
$$

Upper-case Latin subscripts take on the values $0,1,2$, and 3; the repeated subscript $K$ represents a summation from 0 to 3 . The Green's functions in the right-hand side, which are defined in equations A-17 and A-18, represent the observed particle velocities at $\mathbf{x}_{A}$ and $\mathbf{x}_{B}$ due to sources at $\mathbf{x}$ on $\partial \mathrm{D}$. The superscript $\phi$ denotes that these sources are P-wave sources (for $K=0$ ) and $\mathrm{S}$-wave sources with different polarizations (for $K=1,2,3$ ). Hence, the summation over the re- 
peated subscript $K$ represents a summation over P- and S-wave source responses. Note that equation 73 is slightly different from equation 5 in Wapenaar (2004), where the sources of the Green's functions were power-flux normalized. Here we follow a different approach in order to maintain the analogy between the acoustic and elastodynamic expressions (compare equation 73 with equation 29). We postpone power normalization until we introduce noise sources in equation 84

Despite the simple form of equation 73 in comparison with the original equation 63 , this equation still requires the availability of monopole and dipole P- and S-wave source responses. When only monopole responses are available, we have to express the dipole response $\partial_{i} \hat{G}_{p, K}^{v, \phi}\left(\mathbf{x}_{A}, \mathbf{x}, \omega\right) n_{i}$ in terms of the monopole response $\hat{G}_{p, K}^{v, \phi}\left(\mathbf{x}_{A}, \mathbf{x}, \omega\right)$. Note that like the acoustic case, this Green's function obeys Helmholtz equations for $\mathbf{x}$ at and outside $\partial \mathbb{D}$ (see the remark at the end of the Appendix ). Hence, to obtain the dipole responses, each P-wave constituent of the monopole response should be multiplied by $-j \frac{\omega}{c_{P}}|\cos \alpha(\mathbf{x})|$ and each S-wave constituent by $-j \frac{\omega}{c_{S}}|\cos \beta(\mathbf{x})|$, where $\alpha(\mathbf{x})$ and $\beta(\mathbf{x})$ are the local angles between the pertinent $\mathrm{P}$ - and S-rays and the normal on $\partial \mathrm{D}$. However, because $\alpha(\mathbf{x})$ and $\beta(\mathbf{x})$ may have multiple values that are generally unknown, we approximate the dipole response by

$$
\partial_{i} \hat{G}_{p, K}^{v, \phi}\left(\mathbf{x}_{A}, \mathbf{x}, \omega\right) n_{i} \approx-j \frac{\omega}{c^{K}} \hat{G}_{p, K}^{v, \phi}\left(\mathbf{x}_{A}, \mathbf{x}, \omega\right),
$$

with

$$
c^{K}= \begin{cases}c_{P} & \text { for } K=0, \\ c_{S} & \text { for } K=1,2,3 .\end{cases}
$$

Since $K$ is not a subscript in $c^{K}$, no summation takes place over $K$ in the right-hand side of equation 74 . Using equation 74 , equation 73 becomes

$$
\begin{aligned}
2 \Re\left\{\hat{G}_{p, q}^{v, f}\left(\mathbf{x}_{A}, \mathbf{x}_{B}, \omega\right)\right\} \\
\quad \approx \frac{2}{\rho c^{K}} \oint_{\partial \mathrm{D}}\left\{\hat{G}_{p, K}^{v, \phi}\left(\mathbf{x}_{A}, \mathbf{x}, \omega\right)\right\}^{*} \hat{G}_{q, K}^{v, \phi}\left(\mathbf{x}_{B}, \mathbf{x}, \omega\right) \mathrm{d}^{2} \mathbf{x} .
\end{aligned}
$$

The approximation in equation 74 is quite accurate when $\partial \mathrm{D}$ is a sphere with very large radius, since in this case all rays are normal to $\partial \mathrm{D}$ (i.e., $\alpha \approx \beta \approx 0$ ). In general, however, this approximation involves an amplitude error that can be significant. Moreover, spurious events may occur due to incomplete cancellation of contributions from different stationary points. However, because the approximation in equation 74 does not affect the phase of equation 76 , it is considered acceptable for seismic interferometry.

Note that when $\mathbf{x}_{A}$ and $\mathbf{x}_{B}$ are chosen at the free surface $\partial \mathbb{D}_{0}$ (Figure 8), the left-hand sides of equations 73 and 76 should be replaced by $2 \Re\left\{\hat{G}_{p, q}^{v, \tau}\left(\mathbf{x}_{A}, \mathbf{x}_{B}, \omega\right)\right\}$ and the right-hand sides need to be evaluated over $\partial \mathrm{D}_{1}$ only, analogous to equation 72 .

\section{Transient sources}

Until now we assumed that the sources on $\partial \mathbb{D}$ are impulsive point sources. When the sources are transient sources with wavelet $s^{K}(\mathbf{x}, t)$ and corresponding spectrum $\hat{s}^{K}(\mathbf{x}, \omega)$, we write for the observed wavefields at $\mathbf{x}_{A}$ and $\mathbf{x}_{B}$

$$
\begin{aligned}
& \hat{v}_{p, K}^{\mathrm{obs}}\left(\mathbf{x}_{A}, \mathbf{x}, \omega\right)=\hat{G}_{p, K}^{v, \phi}\left(\mathbf{x}_{A}, \mathbf{x}, \omega\right) \hat{s}^{K}(\mathbf{x}, \omega), \\
& \hat{v}_{q, K}^{\mathrm{obs}}\left(\mathbf{x}_{B}, \mathbf{x}, \omega\right)=\hat{G}_{q, K}^{v, \phi}\left(\mathbf{x}_{B}, \mathbf{x}, \omega\right) \hat{s}^{K}(\mathbf{x}, \omega) .
\end{aligned}
$$

Note that $\hat{s}^{K}(\mathbf{x}, \omega)$ is the source spectrum of the P-wave source (for $K=0$ ) and of the S-wave sources with different polarizations (for $K=1,2,3)$. We define the power spectrum of the sources as

$$
\hat{S}^{K}(\mathbf{x}, \omega)=\hat{s}^{K^{*}}(\mathbf{x}, \omega) \hat{s}^{K}(\mathbf{x}, \omega) .
$$

Using these equations, we can modify equation 76 as follows:

$$
\begin{aligned}
2 \mathfrak{R}\left\{\hat{G}_{p, q}^{v, f}\left(\mathbf{x}_{A}, \mathbf{x}_{B}, \omega\right)\right\} \hat{S}_{0}(\omega) \\
\quad \approx \frac{2}{\rho c^{K}} \oint_{\partial \mathrm{D}} \hat{\mathcal{F}}^{K}(\mathbf{x}, \omega) \hat{v}_{p, K}^{\mathrm{obs} *}\left(\mathbf{x}_{A}, \mathbf{x}, \omega\right) \hat{v}_{q, K}^{\mathrm{obs}}\left(\mathbf{x}_{B}, \mathbf{x}, \omega\right) \mathrm{d}^{2} \mathbf{x}
\end{aligned}
$$

where $\hat{S}_{0}(\omega)$ is some average (arbitrarily chosen) power spectrum and $\hat{\mathcal{F}}^{K}(\mathbf{x}, \omega)$ is a shaping filter defined as

$$
\hat{\mathcal{F}}^{K}(\mathbf{x}, \omega)=\frac{\hat{S}_{0}(\omega)}{\hat{S}^{K}(\mathbf{x}, \omega)} .
$$

Equation 80 is well suited for seismic interferometry. It can be applied when a number of natural transient $P$ - and $S$-wave sources with different wavelets on $\partial \mathrm{D}$ radiate wavefields to $\mathbf{x}_{A}$ and $\mathbf{x}_{B}$, that are measured independently for each source and each source-type at $\mathbf{x}$ on $\partial \mathrm{D}$. The shaping filter corrects for the differences in the power spectra of the different sources on $\partial \mathrm{D}$ (this requires that these power spectra are known). Not all sources are equally important: the main contributions to the reconstructed Green's function come from stationary points on $\partial \mathrm{D}$.

\section{Uncorrelated noise sources}

For the transient sources discussed above, we had to assume that the response of each source and each source-type at $\mathbf{x}$ on $\partial \mathbb{D}$ could be measured separately. Here we show that this need is obviated when the sources are mutually uncorrelated noise sources. We define the noise signal for the $K$ th source type at $\mathbf{x}$ on $\partial \mathrm{D}$ as $N_{K}(\mathbf{x}, t)$ and its corresponding spectrum as $\hat{N}_{K}(\mathbf{x}, \omega)$. When all noise sources act simultaneously, we may write for the observed wavefields at $\mathbf{x}_{A}$ and $\mathbf{x}_{B}$

$$
\begin{aligned}
& \hat{v}_{p}^{\mathrm{obs}}\left(\mathbf{x}_{A}, \omega\right)=\oint_{\partial \mathrm{D}} \hat{G}_{p, K}^{v, \phi}\left(\mathbf{x}_{A}, \mathbf{x}, \omega\right) \hat{N}_{K}(\mathbf{x}, \omega) \mathrm{d}^{2} \mathbf{x}, \\
& \hat{v}_{q}^{\mathrm{obs}}\left(\mathbf{x}_{B}, \omega\right)=\oint_{\partial \mathrm{D}} \hat{G}_{q, L}^{v, \phi}\left(\mathbf{x}_{B}, \mathbf{x}^{\prime}, \omega\right) \hat{N}_{L}\left(\mathbf{x}^{\prime}, \omega\right) \mathrm{d}^{2} \mathbf{x}^{\prime} .
\end{aligned}
$$

We assume that two noise sources $\hat{N}_{K}(\mathbf{x}, \omega)$ and $\hat{N}_{L}\left(\mathbf{x}^{\prime}, \omega\right)$ are mutually uncorrelated for any $K \neq L$ and $\mathbf{x} \neq \mathbf{x}^{\prime}$ at $\partial \mathbb{D}$, and that their power spectrum is the same for all $\mathbf{x}$, apart from a power normalization factor $\rho c_{P} / \rho c^{K}$. Hence, we assume that these noise sources obey the relation

$$
\left\langle\hat{N}_{K}^{*}(\mathbf{x}, \omega) \hat{N}_{L}\left(\mathbf{x}^{\prime}, \omega\right)\right\rangle=\frac{\rho c_{P}}{\rho c^{K}} \delta_{K L} \delta\left(\mathbf{x}-\mathbf{x}^{\prime}\right) \hat{S}(\omega),
$$

where $\langle\cdot\rangle$ denotes a spatial ensemble average and $\hat{S}(\omega)$ the power spectrum of the noise. Evaluating the crosscorrelation of the ob- 
served wavefields $\hat{v}_{p}^{\text {obs }}\left(\mathbf{x}_{A}, \omega\right)$ and $\hat{v}_{q}^{\text {obs }}\left(\mathbf{x}_{B}, \omega\right)$, using equations 82-84, yields

$$
\begin{aligned}
& \left\langle\hat{v}_{p}^{\mathrm{obs}^{*}}\left(\mathbf{x}_{A}, \omega\right) \hat{v}_{q}^{\mathrm{obs}}\left(\mathbf{x}_{B}, \omega\right)\right\rangle \\
& \quad=\frac{\rho c_{P}}{\rho c^{K}} \oint_{\partial \mathrm{D}}\left\{\hat{G}_{p, K}^{v, \phi}\left(\mathbf{x}_{A}, \mathbf{x}, \omega\right)\right\}^{*} \hat{G}_{q, K}^{v, \phi}\left(\mathbf{x}_{B}, \mathbf{x}, \omega\right) \hat{S}(\omega) \mathrm{d}^{2} \mathbf{x} .
\end{aligned}
$$

Combining this with equation 76 we obtain

$$
2 \Re\left\{\hat{G}_{p, q}^{v, f}\left(\mathbf{x}_{A}, \mathbf{x}_{B}, \omega\right)\right\} \hat{S}(\omega) \approx \frac{2}{\rho c_{P}}\left\langle\hat{v}_{p}^{\mathrm{obs}^{*}}\left(\mathbf{x}_{A}, \omega\right) \hat{v}_{q}^{\mathrm{obs}}\left(\mathbf{x}_{B}, \omega\right)\right\rangle .
$$

Equation 86 is well suited for application in seismic interferometry. The advantage of equation 86 over equation 80 is that no separate measurements of the responses of all sources at $\partial \mathrm{D}$ are required; these responses can be measured simultaneously, according to equations 82 and 83 . The disadvantage is that no corrections can be made for different power spectra of different sources, like with the shaping filter $\hat{\mathcal{F}}^{K}(\mathbf{x}, \omega)$ in equation 80 .

Finally, note that in the time domain, equation 86 becomes

$$
\begin{aligned}
& \int_{-\infty}^{\infty}\left\{G_{p, q}^{v, f}\left(\mathbf{x}_{A}, \mathbf{x}_{B}, t^{\prime}\right)+G_{p, q}^{v, f}\left(\mathbf{x}_{A}, \mathbf{x}_{B},-t^{\prime}\right)\right\} S\left(t-t^{\prime}\right) \mathrm{d} t^{\prime} \\
& \approx \frac{2}{\rho c_{P}}\left|\int_{-\infty}^{\infty} v_{p}^{\mathrm{obs}}\left(\mathbf{x}_{A}, t^{\prime}\right) v_{q}^{\mathrm{obs}}\left(\mathbf{x}_{B}, t+t^{\prime}\right) \mathrm{d} t^{\prime}\right\rangle
\end{aligned}
$$

According to this equation, the crosscorrelation of the observed particle velocities in the $x_{p}$ - and $x_{q}$-directions at $\mathbf{x}_{A}$ and $\mathbf{x}_{B}$ yields the Green's function for a receiver in the $x_{p}$-direction at $\mathbf{x}_{A}$ and a source in the $x_{q}$-direction at $\mathbf{x}_{B}$, convolved with the autocorrelation of the noise sources. Note the striking resemblance with the retrieval of the Green's tensor in diffuse wavefields in disordered media, as discussed by Campillo and Paul (2003) and Shapiro and Campillo (2004).

\section{CONCLUSIONS}

We have given an overview of acoustic and elastodynamic representations of Green's functions in terms of crosscorrelations of wavefields at two observation points in lossless arbitrary inhomogeneous media. Unlike in many other papers on Green's function retrieval, we have made no assumptions with respect to the diffusivity of the wavefield. We have considered open configurations as well as configurations with a free surface. For the open configurations it is assumed that the wavefields are radiated by sources on an arbitrarily shaped surface that encloses the two observation points. For the situation with a free surface it suffices that sources are available on an open surface that, together with the free surface, forms a surface that encloses the two observation points.

The acoustic and elastodynamic Green's function representations are exact, but not directly suited for application in seismic interferometry. The integrand in both representations consists of a superposition of two correlation products that need to be evaluated separately; moreover, different types of sources are assumed to be available for all source positions on the enclosing surface (scalar monopole and dipole sources in the acoustic case; vectorial force and deformation sources in the elastodynamic case). Last, but not least, the sources are assumed to be impulsive point sources, which does not comply with reality. With a number of approximations, we have simplified the integrand to a single correlation product for a reduced number of source types (monopole sources for both states in the acoustic case; monopole P- and S-wave sources for both states in the elastodynamic case). In practice, not all sources are equally important because the main contributions to the reconstructed Green's functions come from stationary points on the enclosing surface. Finally, we have discussed modifications for the situation of transient sources as well as for uncorrelated noise sources. For the situation of transient sources, we have introduced a shaping filter in the representation integral that corrects for the differences in the power spectra of the different sources (assuming these spectra are known). For the situation of uncorrelated noise sources, the representation integral reduced to a direct crosscorrelation of the recorded wavefields at two observation points, analogous to the methods that retrieve Green's functions from diffuse wavefields in disordered media, or in finite media with an irregular bounding surface.

\section{ACKNOWLEDGMENTS}

This work is supported by the Netherlands Research Centre for Integrated Solid Earth Science (ISES).

\section{APPENDIX}

\section{DERIVATION OF EQUATION 73}

We start our derivation of equation 73 by considering the boundary integral in the right-hand side of equation 48 , which we rewrite as

$$
-\oint_{\partial \mathrm{D}}\left\{\hat{v}_{i, A}^{*} \hat{t}_{i, B}+\hat{t}_{i, A}^{*} \hat{v}_{i, B}\right\} \mathrm{d}^{2} \mathbf{x}
$$

where $\hat{t}_{i, A}=\hat{\tau}_{i j, A} n_{j}$ and $\hat{t}_{i, B}=\hat{\tau}_{i j, B} n_{j}$ are the tractions at $\partial \mathrm{D}$ in states $A$ and $B$. Everything we discuss below also applies to the free-surface configuration of Figure 8, with integration boundary $\partial \mathrm{D}_{1}$. We assume that the medium at and outside $\partial \mathrm{D}$ is homogeneous, isotropic and source-free, with $\mathrm{P}$ - and $\mathrm{S}$-wave propagation velocities $c_{P}$ and $c_{S}$, respectively, and mass density $\rho$. At and outside $\partial \mathrm{D}$ we express the particle velocities in terms of potentials $\hat{\Phi}$ and $\hat{\Psi}_{k}$ for P- and S-waves, according to

$$
\hat{v}_{i}=\frac{-1}{j \omega \rho}\left\{\partial_{i} \hat{\Phi}+\varepsilon_{i j k} \partial_{j} \hat{\Psi}_{k}\right\}, \quad \text { with } \partial_{k} \hat{\Psi}_{k}=0
$$

for states $A$ and $B$. Here $\varepsilon_{i j k}$ is the alternating tensor (or Levi-Civita tensor), with $\varepsilon_{123}=\varepsilon_{312}=\varepsilon_{231}=-\varepsilon_{213}=-\varepsilon_{321}=-\varepsilon_{132}=1$ and the other elements equal to zero. Note that $\hat{\Phi}$ and $\hat{\Psi}_{k}$ obey Helmholtz equations, according to

$$
\partial_{i} \partial_{i} \hat{\Phi}+\left(\omega^{2} / c_{P}^{2}\right) \hat{\Phi}=0
$$

and

$$
\partial_{i} \partial_{i} \hat{\Psi}_{k}+\left(\omega^{2} / c_{S}^{2}\right) \hat{\Psi}_{k}=0
$$

for states $A$ and $B$ (Aki and Richards, 1980). Moreover, note that $\hat{\Phi}$ and $\hat{\Psi}_{k}$ can be explicitly expressed in terms of $\hat{v}_{i}$, according to 


$$
\hat{\Phi}=-\frac{\rho c_{P}^{2}}{j \omega} \partial_{i} \hat{v}_{i}
$$

and

$$
\hat{\Psi}_{k}=\frac{\rho c_{S}^{2}}{j \omega} \varepsilon_{k j i} \partial_{j} \hat{v}_{i}
$$

for states $A$ and $B$ (Wapenaar and Haimé, 1990). Using the stressstrain relation together with equation $\mathrm{A}-2$, the traction $\hat{t}_{i}$ can also be expressed in terms of the potentials $\hat{\Phi}$ and $\hat{\Psi}_{l}$. Substituting the result together with equation A-2 for states $A$ and $B$ into equation A-1, we get terms containing $\left\{\hat{\Phi}_{A}^{*}, \hat{\Phi}_{B}\right\}, \quad\left\{\hat{\Phi}_{A}^{*}, \hat{\Psi}_{l, B}\right\}, \quad\left\{\hat{\Psi}_{k, A}^{*}, \hat{\Phi}_{B}\right\}$, and $\left\{\hat{\Psi}_{k, A}^{*}, \hat{\Psi}_{l, B}\right\}$. Wapenaar and Haimé (1990) analyze this substitution for the situation of a horizontal boundary $S_{1}$ with a downward pointing normal vector $\mathbf{n}=(0,0,1)$. When all wavefields at $S_{1}$ are propagating downward, this substitution leads to

$$
\begin{aligned}
& -\int_{S_{1}}\left\{\hat{v}_{i, A}^{*} \hat{t}_{i, B}+\hat{t}_{i, A}^{*} \hat{v}_{i, B}\right\} \mathrm{d}^{2} \mathbf{x} \\
& =\frac{2}{j \omega \rho} \int_{S_{1}}\left\{\left(\partial_{3} \hat{\Phi}_{A}^{*}\right) \hat{\Phi}_{B}+\left(\partial_{3} \hat{\Psi}_{k, A}^{*}\right) \hat{\Psi}_{k, B}\right\} \mathrm{d}^{2} \mathbf{x} .
\end{aligned}
$$

Note that the right-hand side of this equation does not contain products of $\mathrm{P}$-waves in one state and $\mathrm{S}$-waves in the other and vice versa. Equation A-7 was derived under the assumption that evanescent waves may be neglected. This implies that it does not account for horizontally propagating wave modes like surface waves. We will now discuss how equation A-7 can be modified for the closed surface $\partial \mathrm{D}$ and argue that this modified result does account for surface waves.

In the high frequency regime, the main contributions to the integral in equation A-1 come from points on $\partial \mathbb{D}$ where the integrand is stationary. Upon substitution of the P- and S-wave potentials for states $A$ and $B$, different types of stationary points occur. For the term containing $\left\{\hat{\Phi}_{A}^{*}, \hat{\Phi}_{B}\right\}$, the integrand is stationary at those points on $\partial \mathrm{D}$ where the ray angles $\alpha_{A}$ and $\alpha_{B}$ of the P-waves in both states are identical. Similarly, for the term containing $\left\{\hat{\Psi}_{k, A}^{*}, \hat{\Psi}_{l, B}\right\}$ the stationary points occur where the ray angles $\beta_{A}$ and $\beta_{B}$ of the $S$-waves in both states are identical. The stationary points for the term containing $\left\{\hat{\Phi}_{A}^{*}, \hat{\Psi}_{l, B}\right\}$ are determined by the condition $\left(\sin \alpha_{A}\right) / c_{P}=\left(\sin \beta_{B}\right) / c_{S}$. Finally, the term containing $\left\{\hat{\Psi}_{k, A}^{*}, \hat{\Phi}_{B}\right\}$ is stationary at those points on $\partial \mathrm{D}$ where $\left(\sin \beta_{A}\right) / c_{S}=\left(\sin \alpha_{B}\right) / c_{P}$. At each stationary point we choose a local coordinate system, with the $x_{3}$-axis parallel to the local outward pointing normal on $\partial \mathrm{D}$. Note that the inner products $\hat{v}_{i, A}^{*} \hat{t}_{i, B}$ and $\hat{t}_{i, A}^{*} \hat{v}_{i, B}$ of the integrand in equation A-1 remain inner products in the local coordinate system. Hence, it is justified to substitute equation A-2 in the local coordinate system into equation A-1 and apply a similar analysis as in Wapenaar and Haimé (1990) in the local coordinate system, assuming all waves are propagating outward at $\partial \mathrm{D}$. Depending on the type of stationary point this leads to contributions of the form $\left(\partial_{3} \hat{\Phi}_{A}^{*}\right) \hat{\Phi}_{B}$ or $\left(\partial_{3} \hat{\Psi}_{k, A}^{*}\right) \hat{\Psi}_{k, B}$ in the local coordinate system; at those stationary points where $\left(\sin \alpha_{A}\right) / c_{P}=\left(\sin \beta_{B}\right) / c_{S}$ or where $\left(\sin \beta_{A}\right) / c_{S}=\left(\sin \alpha_{B}\right) / c_{P}$ the terms cancel. The inner products $\left(\partial_{3} \hat{\Psi}_{k, A}^{*}\right) \hat{\Psi}_{k, B}$ in the local coordinate system remain inner products in the absolute coordinate system; only the derivative $\partial_{3}$ in the local system has to be replaced by $n_{i} \partial_{i}$ in the absolute system. Hence, when we apply the outlined procedure for all stationary points on $\partial \mathrm{D}$, we finally obtain (in the absolute coordinate system)

$$
\begin{aligned}
& -\oint_{\partial \mathrm{D}}\left\{\hat{v}_{i, A}^{*} \hat{t}_{i, B}+\hat{t}_{i, A}^{*} \hat{v}_{i, B}\right\} \mathrm{d}^{2} \mathbf{x} \\
& =\frac{2}{j \omega \rho} \oint_{\partial \mathrm{D}}\left\{\left(\partial_{i} \hat{\Phi}_{A}^{*}\right) \hat{\Phi}_{B}+\left(\partial_{i} \hat{\Psi}_{k, A}^{*}\right) \hat{\Psi}_{k, B}\right\} n_{i} \mathrm{~d}^{2} \mathbf{x} .
\end{aligned}
$$

In equation A-7, horizontally propagating wave modes, like surface waves, were excluded, which was a consequence of choosing a horizontal integration boundary. Another way of explaining this is that the horizontal integration boundary does not contain the stationary points that would contribute to the surface waves. For the closed boundary integral of equation A-8, horizontally propagating modes are no more exclusive than any other wave type propagating in any direction. Hence, equation A-8 accounts for surface waves; the main stationary points for these waves are located somewhere at the sides of $\partial \mathrm{D}$.

Substituting equation A-8 into the right-hand side of equation 48 gives

$$
\begin{gathered}
\int_{\mathrm{D}}\left\{-\hat{\tau}_{i j, A}^{*} \hat{h}_{i j, B}+\hat{v}_{i, A}^{*} \hat{f}_{i, B}-\hat{h}_{i j, A}^{*} \hat{\tau}_{i j, B}+\hat{f}_{i, A}^{*} \hat{v}_{i, B}\right\} \mathrm{d}^{3} \mathbf{x} \\
=\frac{2}{j \omega \rho} \oint_{\partial \mathrm{D}}\left\{\left(\partial_{i} \hat{\Phi}_{A}^{*}\right) \hat{\Phi}_{B}+\left(\partial_{i} \hat{\Psi}_{k, A}^{*}\right) \hat{\Psi}_{k, B}\right\} n_{i} \mathrm{~d}^{2} \mathbf{x} .
\end{gathered}
$$

We choose point sources of force at $\mathbf{x}_{A}$ and $\mathbf{x}_{B}$ (equations 51 and 52), whereas the deformation sources are chosen equal to zero. For $\mathbf{x}$ in $\mathbb{D}$, the velocities and stresses in states $A$ and $B$ are again expressed in terms of elastodynamic Green's functions, according to equations 53-56. For $\mathbf{x}$ at and outside $\partial \mathrm{D}$, the $\mathrm{P}$ - and $\mathrm{S}$-wave potentials in states $A$ and $B$ are expressed in terms of Green's functions, according to

$$
\begin{array}{r}
\hat{\Phi}_{A}(\mathbf{x}, \omega) \triangleq \hat{G}_{0, p}^{\phi, f}\left(\mathbf{x}, \mathbf{x}_{A}, \omega\right), \\
\hat{\Psi}_{k, A}(\mathbf{x}, \omega) \triangleq \hat{G}_{k, p}^{\phi, f}\left(\mathbf{x}, \mathbf{x}_{A}, \omega\right), \\
\hat{\Phi}_{B}(\mathbf{x}, \omega) \triangleq \hat{G}_{0, q}^{\phi, f}\left(\mathbf{x}, \mathbf{x}_{B}, \omega\right), \\
\hat{\Psi}_{k, B}(\mathbf{x}, \omega) \triangleq \hat{G}_{k, q}^{\phi, f}\left(\mathbf{x}, \mathbf{x}_{B}, \omega\right) .
\end{array}
$$

The superscript $\phi$ denotes that the observed wavefield quantity at $\mathbf{x}$ is a $\mathrm{P}$ - or $\mathrm{S}$-wave potential. To describe both wave types with one Green's function, we introduce an upper-case Latin subscript $K$ that takes on the values $0,1,2$, and 3 . Hence, in $\hat{G}_{K, p}^{\phi, f}\left(\mathbf{x}, \mathbf{x}_{A}, \omega\right)$ the observed wavefield at $\mathbf{x}$ is a $\mathrm{P}$-wave (for $K=0$ ) or an S-wave component (for $K=k=1,2,3$ ), respectively. Substituting equations 51-53, 55, and A-10-A-13 into equation A-9 gives

$$
\begin{aligned}
& \left\{\hat{G}_{q, p}^{v, f}\left(\mathbf{x}_{B}, \mathbf{x}_{A}, \omega\right)\right\}^{*}+\hat{G}_{p, q}^{v, f}\left(\mathbf{x}_{A}, \mathbf{x}_{B}, \omega\right) \\
& \quad=\frac{2}{j \omega \rho} \oint_{\partial \mathrm{D}}\left\{\partial_{i} \hat{G}_{K, p}^{\phi, f}\left(\mathbf{x}, \mathbf{x}_{A}, \omega\right)\right\}^{*} \hat{G}_{K, q}^{\phi, f}\left(\mathbf{x}, \mathbf{x}_{B}, \omega\right) n_{i} \mathrm{~d}^{2} \mathbf{x} .
\end{aligned}
$$

The repeated subscript $K$ represents a summation from 0 to 3 and thus accounts for the summation of the different wave types in equation A-9. 
Note that $\hat{G}_{K, p}^{\phi, f}\left(\mathbf{x}, \mathbf{x}_{A}, \omega\right)$ can be expressed in terms of $\hat{G}_{i, p}^{v, f}(\mathbf{x}$, $\left.\mathbf{x}_{A}, \omega\right)$. Using equations 53, A-5, A-6, A-10, and A-11, we obtain

$$
\hat{G}_{0, p}^{\phi, f}\left(\mathbf{x}, \mathbf{x}_{A}, \omega\right)=-\frac{\rho c_{P}^{2}}{j \omega} \partial_{i} \hat{G}_{i, p}^{v, f}\left(\mathbf{x}, \mathbf{x}_{A}, \omega\right)
$$

and

$$
\hat{G}_{k, p}^{\phi, f}\left(\mathbf{x}, \mathbf{x}_{A}, \omega\right)=\frac{\rho c_{S}^{2}}{j \omega} \varepsilon_{k j i} \partial_{j} \hat{G}_{i, p}^{v, f}\left(\mathbf{x}, \mathbf{x}_{A}, \omega\right)
$$

(and similar expressions for state $B$ ). We define reciprocal Green's functions, analogous to equations A-15 and A-16, as

$$
\hat{G}_{p, 0}^{v, \phi}\left(\mathbf{x}_{A}, \mathbf{x}, \omega\right) \triangleq-\frac{\rho c_{P}^{2}}{j \omega} \partial_{i} \hat{G}_{p, i}^{v, f}\left(\mathbf{x}_{A}, \mathbf{x}, \omega\right)
$$

and

$$
\hat{G}_{p, k}^{v, \phi}\left(\mathbf{x}_{A}, \mathbf{x}, \omega\right) \triangleq \frac{\rho c_{S}^{2}}{j \omega} \varepsilon_{k j i} \partial_{j} \hat{G}_{p, i}^{v, f}\left(\mathbf{x}_{A}, \mathbf{x}, \omega\right)
$$

(and similar definitions for state $B$ ). Note that the differentiation operators at the right-hand sides of these equations act on the source coordinate $\mathbf{x}$. The superscript $\phi$ now denotes that the source at $\mathbf{x}$ is a source for P- or S-waves. Hence, in $\hat{G}_{p, K}^{v, \phi}\left(\mathbf{x}_{A}, \mathbf{x}, \omega\right)$ the source is a source for P-waves (for $K=0$ ) or for S-waves with different polarizations (for $K=k=1,2,3$ ), respectively.

On account of equations 60 and A-15 and A-18 the following reciprocity relation is obtained

$$
\hat{G}_{K, p}^{\phi, f}\left(\mathbf{x}, \mathbf{x}_{A}, \omega\right)=\hat{G}_{p, K}^{v, \phi}\left(\mathbf{x}_{A}, \mathbf{x}, \omega\right) .
$$

Similarly,

$$
\hat{G}_{K, q}^{\phi, f}\left(\mathbf{x}, \mathbf{x}_{B}, \omega\right)=\hat{G}_{q, K}^{v, \phi}\left(\mathbf{x}_{B}, \mathbf{x}, \omega\right) .
$$

Using reciprocity relations $60, \mathrm{~A}-19$, and A-20 in equation A-14 we obtain equation 73 .

Finally, note that according to equations A-3, A-4, A-10-A-13, A-19, and A-20, the Green's functions in the right-hand side of equation 73 obey Helmholtz equations for $\mathbf{x}$ at and outside $\partial \mathbb{D}$, similar as the acoustic Green's functions in the right-hand side of equation 29.

\section{REFERENCES}

Aki, K., and P. G. Richards, 1980, Quantitative seismology, vol. 1: W. H. Freeman and Company.

Bleistein, N., 1984, Mathematical methods for wave phenomena: Academic Press Inc.

Bojarski, N. N., 1983, Generalized reaction principles and reciprocity theorems for the wave equations, and the relationship between the time-advanced and time-retarded fields: Journal of the Acoustical Society of America, 74, 281-285.

Campillo, M., and A. Paul, 2003, Long-range correlations in the diffuse seismic coda: Science, 299, 547-549.

Claerbout, J. F., 1968, Synthesis of a layered medium from its acoustic transmission response: Geophysics, 33, 264-269.

de Hoop, A. T., 1966, An elastodynamic reciprocity theorem for linear, viscoelastic media: Applied Scientific Research, 16, 39-45.

_ 1988, Time-domain reciprocity theorems for acoustic wave fields in fluids with relaxation: Journal of the Acoustical Society of America, 84, $1877-1882$.
Inc

Derode, A., E. Larose, M. Campillo, and M. Fink, 2003a, How to estimate the Green's function of a heterogeneous medium between two passive sensors? Application to acoustic waves: Applied Physics Letters, 83, 3054-3056.

Derode, A., E. Larose, M. Tanter, J. de Rosny, A. Tourin, M. Campillo, and M. Fink, 2003b, Recovering the Green's function from field-field correlations in an open scattering medium (L): Journal of the Acoustical Society of America, 113, 2973-2976.

Draganov, D., K. Wapenaar, and J. Thorbecke, 2003, Synthesis of the reflection response from the transmission response in the presence of white noise sources: 65th Annual International Meeting, EAGE, Extended Abstracts, Session P218.

- 2006, Seismic interferometry: Reconstructing the earth's reflection response: Geophysics, this issue.

Fink, M., 1997, Time reversed acoustics: Physics Today, 50, 34-40.

Fokkema, J. T., and P. M. van den Berg, 1993, Seismic applications of acoustic reciprocity: Elsevier Science Publishing Company, Inc.

Knopoff, L., and A. F. Gangi, 1959, Seismic reciprocity: Geophysics, 24 681-691.

Lobkis, O. I., and R. L. Weaver, 2001, On the emergence of the Green's function in the correlations of a diffuse field: Journal of the Acoustical Society of America, 110, 3011-3017.

Malcolm, A. E. J. A. Scales, and B. A. van Tiggelen, 2004, Extracting the Green function from diffuse, equipartitioned waves: Physical Review E, 70, 015601-1-015601-4.

Pao, Y. H., and V. Varatharajulu, 1976, Huygens' principle, radiation conditions, and integral formulations for the scattering of elastic waves: Journal of the Acoustical Society of America, 59, 1361-1371.

Rayleigh, J. W. S., 1878, The theory of sound, Vol. II: Dover Publications Inc. (Reprint 1945).

Roux, P., K. G. Sabra, W. A. Kuperman, and A. Roux, 2005, Ambient noise cross correlation in free space: Theoretical approach: Journal of the Acoustical Society of America, 117, 79-84.

Schuster, G. T., 2001, Theory of daylight/interferometric imaging: tutorial: 63rd Meeting, European Association of Geoscientists and Engineers, Extended Abstracts, Session A32.

Schuster, G. T., J. Yu, J. Sheng, and J. Rickett, 2004, Interferometric/daylight seismic imaging: Geophysical Journal International, 157, 838-852.

Shapiro, N. M., and M. Campillo, 2004, Emergence of broadband Rayleigh waves from correlations of the ambient seismic noise: Geophysical Research Letters, 31, L07614-1-L07614-4.

Shapiro, N. M., M. Campillo, L. Stehly, and M. H. Ritzwoller, 2005, Highresolution surface-wave tomography from ambient seismic noise: Science, 307, 1615-1618.

Snieder, R., 2004, Extracting the Green's function from the correlation of coda waves: A derivation based on stationary phase: Physical Review E, 69,046610-1-046610-8.

Snieder, R., K. Wapenaar, and K. Larner, 2006, Spurious multiples in seismic interferometry of primaries: Geophysics, this issue.

van Manen, D.-J., J. O. A. Robertsson, and A. Curtis, 2005, Modeling of wave propagation in inhomogeneous media: Physical Review Letters, 94, 164301-1-164301-4.

van Tiggelen, B. A., 2003, Green function retrieval and time reversal in a disordered world: Physical Review Letters, 91, 243904-1-243904-4.

Wapenaar, K., 2004, Retrieving the elastodynamic Green's function of an arbitrary inhomogeneous medium by cross correlation: Physical Review Letters, 93, 254301-1-254301-4.

Wapenaar, K., D. Draganov, J. Thorbecke, and J. Fokkema, 2002, Theory of acoustic daylight imaging revisited: 72nd Annual International Meeting, SEG, Expanded Abstracts, 2269-2272.

Wapenaar, K., D. Draganov, J. van der Neut, and J. Thorbecke, 2004a, Seismic interferometry: a comparison of approaches: 74th Annual International Meeting, SEG, Expanded Abstracts, 1981-1984.

Wapenaar, K., J. Thorbecke, and D. Draganov, 2004b, Relations between reflection and transmission responses of three-dimensional inhomogeneous media: Geophysical Journal International, 156, 179-194.

Wapenaar, C. P. A., and G. C. Haimé, 1990, Elastic extrapolation of primary seismic P- and S-waves: Geophysical Prospecting, 38, 23-60.

Weaver, R. L., and O. I. Lobkis, 2001, Ultrasonics without a source: Thermal fluctuation correlations at MHz frequencies: Physical Review Letters, 87, 134301-1-134301-4.

, 2002, On the emergence of the Green's function in the correlations of a diffuse field: pulse-echo using thermal phonons: Ultrasonics, 40 , $435-439$.

,- 2004 , Diffuse fields in open systems and the emergence of the Green's function (L): Journal of the Acoustical Society of America, 116, 2731-2734. 